Review Article

\title{
Immunoregulation of Shenqi Fuzheng Injection Combined with Chemotherapy in Cancer Patients: A Systematic Review and Meta-Analysis
}

\author{
Yang Yang, ${ }^{1}$ Wang Ting, ${ }^{1}$ Liu Xiao, ${ }^{1}$ Fu Shufei, ${ }^{1}$ Tan Wangxiao, ${ }^{1}$ Wang Xiaoying, ${ }^{1,2}$ \\ Gao Xiumei, ${ }^{1}$ and Zhang Boli ${ }^{1}$ \\ ${ }^{1}$ State Key Laboratory of Modern Chinese Medicine, Tianjin University of Traditional Chinese Medicine, Tianjin 300193, China \\ ${ }^{2}$ College of Traditional Chinese Medicine, Tianjin University of Traditional Chinese Medicine, Tianjin 300193, China
}

Correspondence should be addressed to Wang Xiaoying; wxy@tjutcm.edu.cn and Zhang Boli; zhangbolipr@163.com

Received 23 March 2016; Revised 15 July 2016; Accepted 28 July 2016; Published 5 January 2017

Academic Editor: Gioacchino Calapai

Copyright ( 2017 Yang Yang et al. This is an open access article distributed under the Creative Commons Attribution License, which permits unrestricted use, distribution, and reproduction in any medium, provided the original work is properly cited.

Background. Immunosuppression is a well-recognised complication of chemotherapy in cancer patients. We assemble the clinical evidence that SQI, an adjuvant drug for lung cancer and gastric cancer which was widely prescribed in China, interventions could increase objective tumour response and regulate immunity in cancer patients undergoing chemotherapy. Methods. We undertook a systemic review of the clinical data from randomised controlled trials up to September 2015 in which a SQI intervention was compared with a control arm in patients undergoing conventional chemotherapy. Revman 5.0 Software was used for the data analysis. Results. 49 randomised controlled trials were included in the systematic review. The meta-analysis results demonstrated that the SQI intervention with conventional chemotherapy exhibited better therapeutic efficacy than the conventional chemotherapy group with a statistically significant higher objective tumour response. Cotreatment with SQI could enhance NK, $\mathrm{CD}_{3}{ }^{+}, \mathrm{CD}_{4}{ }^{+}$level, and $\mathrm{CD}_{4}{ }^{+} / \mathrm{CD}_{8}{ }^{+}$ratio comparing with the conventional chemotherapy group. Conclusions. The conclusions of this review might suggest a high risk of bias due to the low quality and the limitation of cancer types in the included trials. A more reliable conclusion regarding the immunoregulation of SQI could be reached based on more trials of higher quality.

\section{Introduction}

The prevalence of cancer continues to increase globally. Although the mortality of cancer has been reduced through advances in treatment such as chemotherapy, the adverse reactions caused by chemotherapy such as cardiotoxicity, myelosuppression, and immunosuppression have increased [1]. It has been increasingly recognised that alternative medicines might be another strategy, and western medicines might not be the only answer while these issues remain unsolved [2-5].

Shenqi Fuzheng injection (SQI) is an injection comprised of Codonopsis pilosula (Franch) Nannf. and Astragalus membranaceus (Fisch.) Bunge [6] and was approved by the State Food and Drug Administration of the People's Republic of China (SFDA) in 1999. As an adjuvant drug for lung cancer and gastric cancer, its efficacy is shown in tonifying qi and strengthening the body's resistance. Researches indicated that SQI could improve the peripheral blood $\mathrm{T}$ cell subsets, promote macrophage proliferation, and alleviate immunosuppression caused by chemotherapy [7, 8]. Currently, there are many published trials about SQI combined with chemotherapy for the treatment of cancers; some of these trials have shown that SQI could improve tumour response and increase immunity indicators [7-10]. However, little is known about SQI outside of China, and there has not been a systematic evaluation on its effects on immunity until now. The hypothesis of this paper was SQI, an adjuvant drug for lung cancer and gastric cancer which was widely prescribed in China, could make a critical difference in alleviating chemotherapy-associated immunosuppression.

This paper presents a systematic review in an effort to clarify if SQI in combination with conventional chemotherapy for 
cancer patients increases the objective tumour response and relative immunity parameters.

\section{Materials and Methods}

2.1. Search Strategy. According to guidelines from the Cochrane collaboration [11], a literature search of PubMed, CNKI (China national knowledge infrastructure, http://www.cnki.net/), VIP (Chongqing VIP Information Co., Ltd, http://www.cqvip .com/), and Wanfang (http://www.wanfangdata.com.cn/) from 1999 (SQI launch) to September 2015 was performed. The search strategy " $((($ cancer $)$ OR tumour $))$ AND shenqi fuzheng injection) AND immune" was adapted for each database. Papers were limited to clinical research in Chinese or English.

2.2. Inclusion and Exclusion Criteria. The studies were included if (1) the study was a randomised controlled trial comparing a SQI plus chemotherapy treatment group with a chemotherapy control group; (2) the patients were diagnosed as having cancer with the age, gender, race, cancer type, and pathological classification and chemotherapy regimens were unlimited; (3) the invention was SQI intravenous drip infusion on the basis of conventional chemotherapy adopted by the control group; the initial time, dosage, and course of medicine treatment were unrestricted; (4) studies contained at least one of the following clinical data points: objective tumour response (the 4-point WHO scale was adopted [12]), natural killer cell $(\mathrm{NK})$, matured $\mathrm{T}$ lymphocytes $\left(\mathrm{CD}_{3}{ }^{+}\right)$, inducer lymphocyte/helper $\mathrm{T}$ lymphocyte $\left(\mathrm{CD}_{4}{ }^{+}\right)$, suppressor T cell/cytotoxic T cell $\left(\mathrm{CD}_{8}^{+}\right)$level, and $\mathrm{CD}_{4}{ }^{+} / \mathrm{CD}_{8}{ }^{+}$ratio; (5) the reported data included estimated relative risk (RR) and $95 \%$ confidence intervals (CIs) for each outcome; (6) in the case of duplicate publications, the maximum sample size version was included.

Studies were excluded if they met any of the following criteria: (1) the studies were case series, case reports, or clinical reports concerning radiotherapy or surgery; (2) the paper used SQI in combination with other herbal medicines or chemical drugs; (3) the articles exhibited no outcomes concerning objective tumour response and immunity index or were presented as an abstract only.

2.3. Data Extraction and Methodological Quality Assessment. Data were independently extracted by two reviewers (Y. Y. and W. T.) using a data collection table. All discrepancies were resolved by consensus. For the systematic review, all data on patient characteristics (number, gender, age, and oncological category), treatment and invention details (chemotherapy regimens, schedule, and course of SQI invention), and clinical outcomes were extracted. The following outcomes were extracted: objective tumour response and immunity indicators including $\mathrm{NK}, \mathrm{CD}_{3}{ }^{+}, \mathrm{CD}_{4}{ }^{+}$, and $\mathrm{CD}_{8}{ }^{+}$levels, and $\mathrm{CD}_{4}{ }^{+} / \mathrm{CD}_{8}{ }^{+}$ratio. The quality of the studies included in the analysis was assessed independently by two reviewers (Y. Y. and W. T.). The methodological quality of the studies was assessed using the modified Jadad scale, an instrument developed and validated to assess the quality of clinical trials by evaluating randomization, blinding, withdrawals/dropouts, and randomization concealment $[13,14]$.
2.4. Data Synthesis and Statistical Analysis. Heterogeneity between studies was assessed by measuring inconsistency $\left(I^{2}\right)$. When $I^{2}<50 \%$, the fixed-effects model was used to calculate the relative ratio (RR) and the $95 \%$ confidence intervals (CIs). Otherwise, a random-effects model was used [15]. The publication bias was examined by using funnel plots. A forest plot was built to show the overall effect of the intervention against control. Statistical analyses were performed using RevMan 5.0 (Cochrane Information Management System, Oxford, United Kingdom (UK)) [11], and $P<0.05$ was considered statistically significant.

\section{Results}

3.1. Description of Studies. A total of 415 studies were identified through the search of databases. 251 studies were retained after the first screening based on the title and abstract. A total of 131 studies were excluded according to the inclusion and exclusion criteria. Among the studies that were retained, 73 randomised controlled trials were selected after fulltext assessment. Forty-nine of the 73 studies were classified into three main categories: 20 trials of lung cancer [1634], 23 trials of digestive tract cancer [35-56], and 6 trials of breast cancer [8,57-61] as shown in Figure 1. For lung cancer, 20 trials included 1597 patients with a median age ranging from 43 to 66.5. A dominance of non-small cell lung cancer existed $(18 / 20,90 \%)$, and the small cell lung cancer accounted for $10 \%$. Platinum-based chemotherapy represented by paclitaxel plus cisplatin was the primary chemotherapy $(10 / 20,50 \%)$. Other chemotherapy regimens contain vinorelbine plus cisplatin, gemcitabine plus cisplatin, and docetaxel plus cisplatin. Regarding digestive tract cancer, 23 studies consisted of 1656 patients with the median age range of 45 to 65.9. Colon cancer, colorectal cancer, gastric cancer, gastrointestinal cancer, and oesophageal cancer were all included in digestive tract cancer. Oxaliplatin and 5-Fu based chemotherapy regiments were widely used in clinic. Six articles were focused on breast cancer with 1656 female patients in a median age of 42 to 56.1. Anthracycline-based chemotherapy was the conventional chemotherapeutic agent. According to the modified Jadad scale [14], the methodology of all studies was low quality with a quality score of 3 or under 3. All the clinical details of the 49 included studies were listed in Table 1. The remaining 24 studies which included 10 kinds of cancers like leukaemia, cervical cancer, and ovarian cancer were not included in the meta-analysis because of the lack of samples.

3.2. Safety Evaluation of Combination Medication of SQI and Chemotherapy. All articles included in the meta-analysis evaluated the safety of the combination medication of SQI and chemotherapy regiments. Detailed safety evaluation information on the combination medication of SQI and chemotherapy agents showed in Table 2. The conclusion could be drawn from the table that gastrointestinal reactions and routine blood indexes decreases were the primary and most mentioned phenomena. 


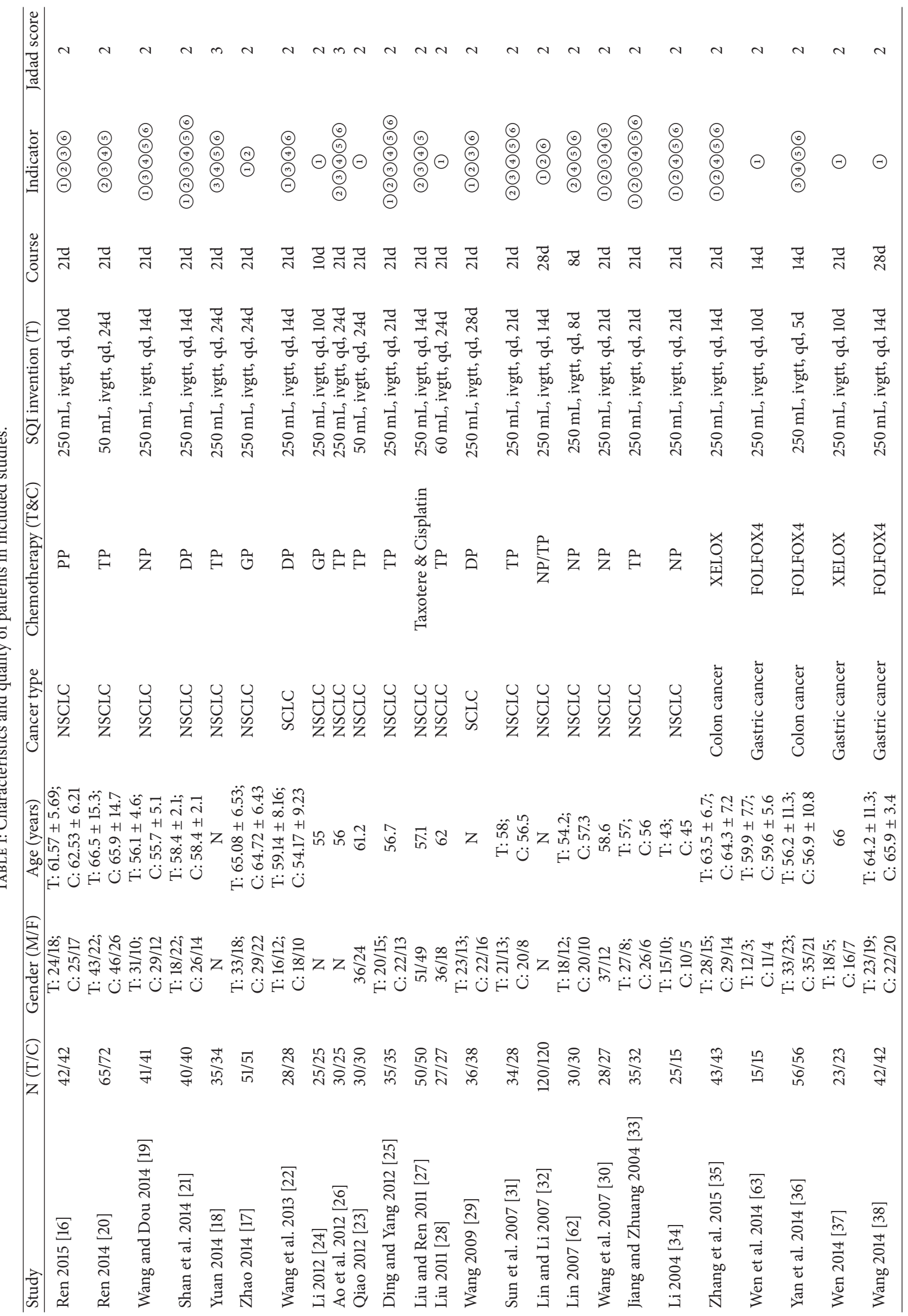




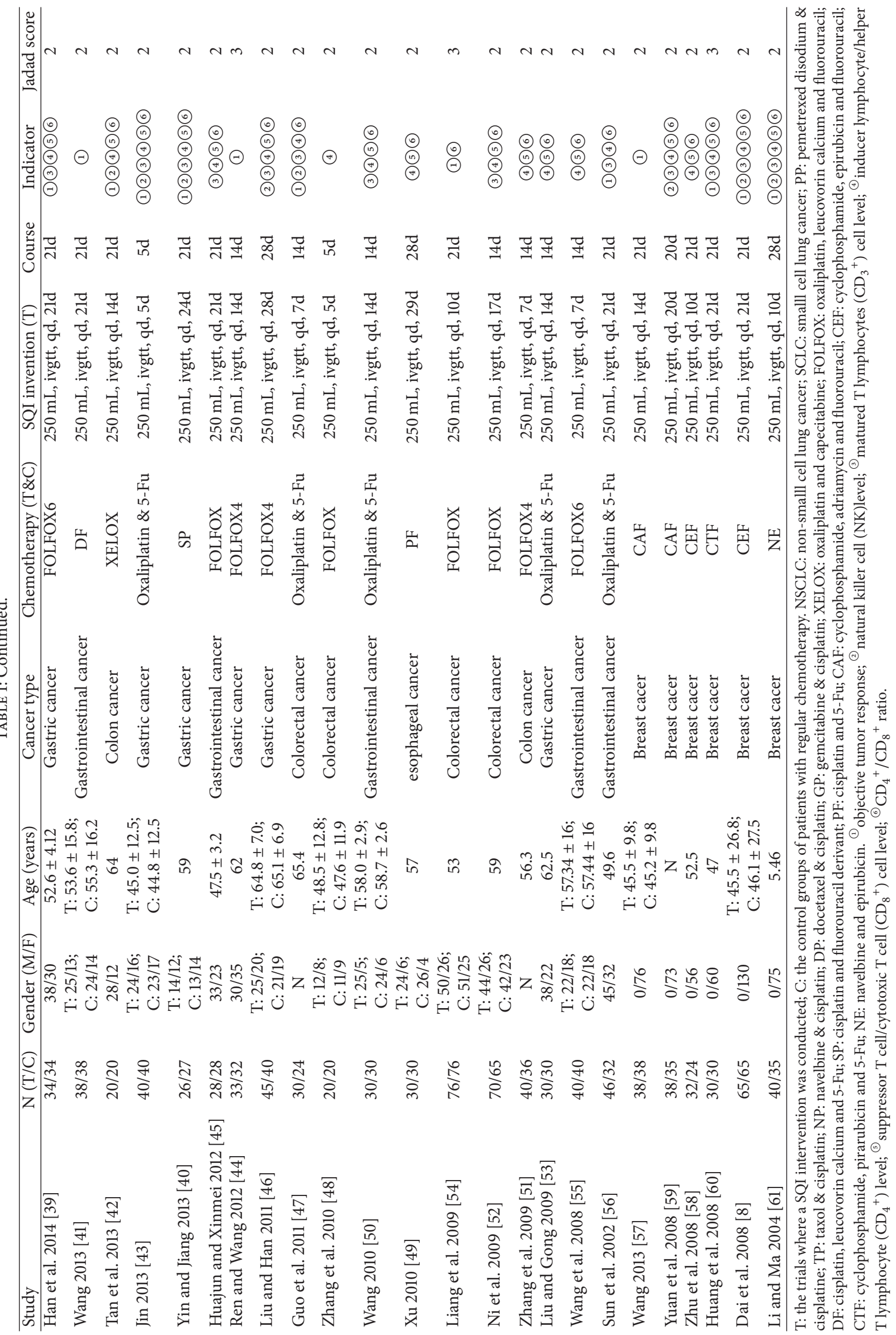




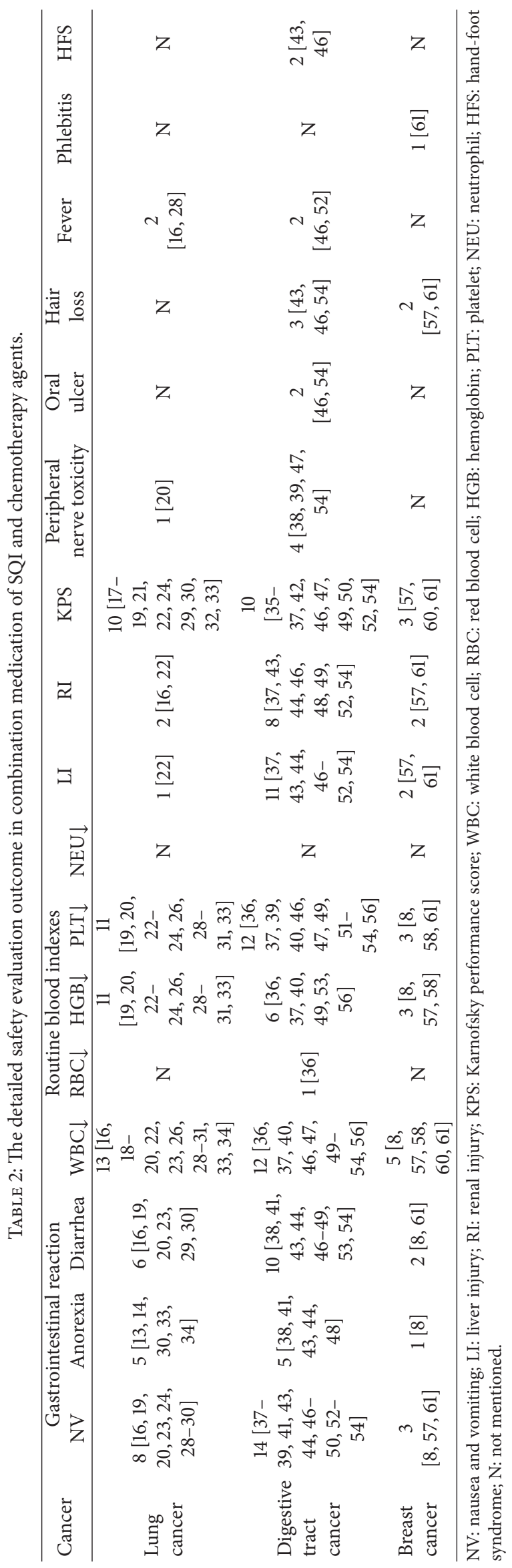




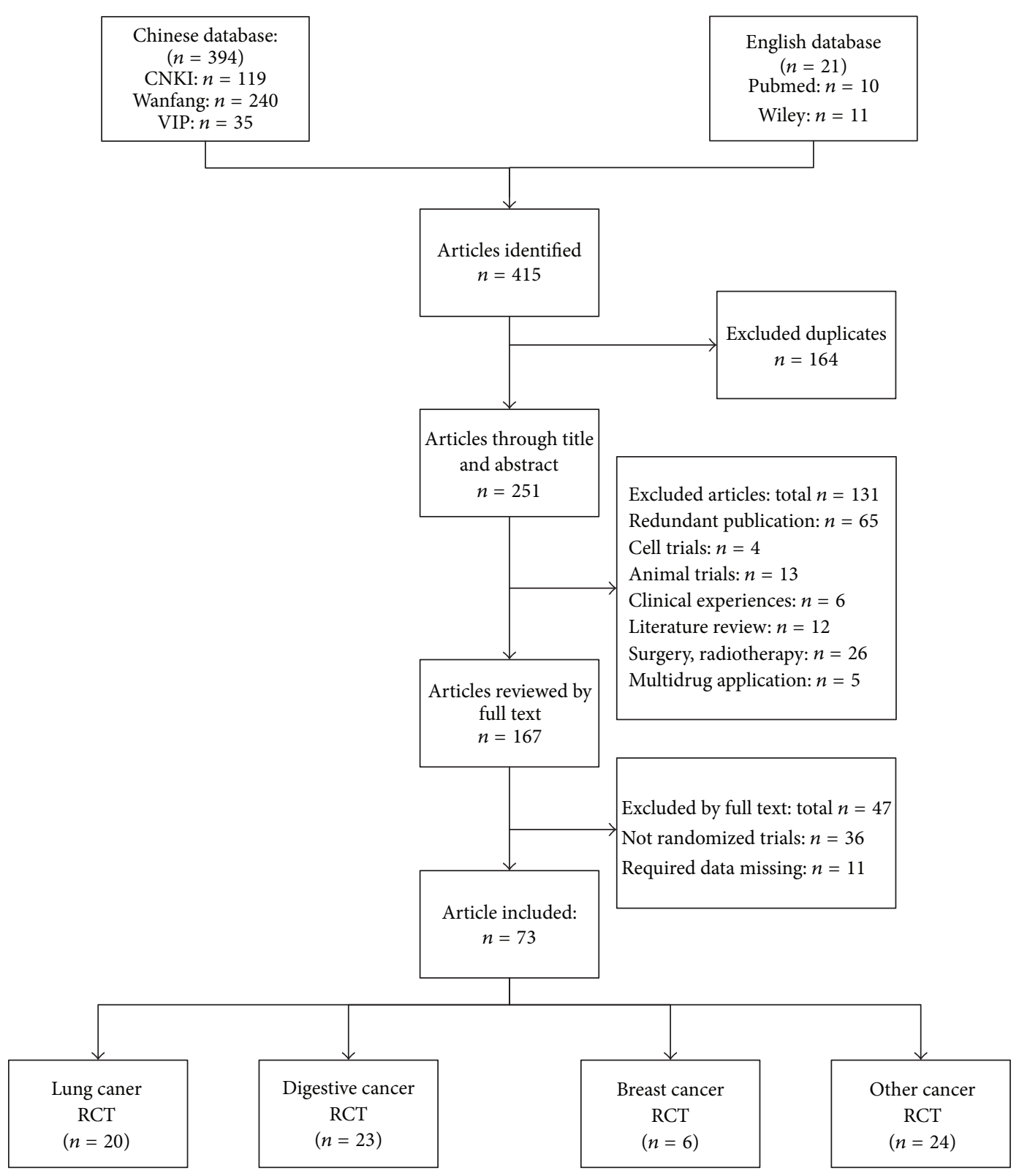

FIGURE 1: Flow chart of literature screening.

3.3. The Results of Meta-Analysis for Clinical Outcomes in Lung Cancer Patients: Objective Tumour Response and Immunity Indicators. In 12 clinical trials concerning objective tumour response in lung cancer patients $[16,17,19,21-25,28-30,34]$, there were 406 patients in the SQI intervention group and 399 patients with conventional chemotherapy in the control group. The results showed that the objective tumour response in the SQI intervention group was better than in the control group $(\mathrm{RR}=1.28,95 \% \mathrm{CI} 1.09-1.49, P=0.002)$.

According to 14 clinical trials [16-18, 20, 21, 25-27, 29-31, $33,34]$ including 536 patients in the SQI intervention group and 519 lung cancer patients with conventional chemotherapy as control group, the NK levels were significant improved by SQI intervention $(\mathrm{RR}=7.64,95 \%$ CI 5.17-10.11, $P<$
0.00001). In 14 clinical trials [16, 18-22, 25-27, 29-31, 33] including 529 patients in the SQI intervention group and 522 patients with conventional chemotherapy, $\mathrm{CD}_{3}{ }^{+}$cell levels were dramatically improved by SQI $(\mathrm{RR}=12.23,95 \% \mathrm{CI}$ 6.56-17.90, $P<0.0001)$. For the $\mathrm{CD}_{4}{ }^{+}$cell levels in lung cancer, there were 14 clinical trials [16, 18-22, 25-27, 30, 31, 33,34 ] including 518 patients in the SQI intervention and 499 patients with conventional chemotherapy. SQI intervention preceded the control group in improving the $\mathrm{CD}_{4}{ }^{+}$cell levels with $\mathrm{RR}=9.99,95 \%$ CI 6.00-13.97, $P<0.0001$. There were 12 clinical trials $[16,18,19,21,22,25,26,29$, $31,33,34]$ including 411 patients in SQI intervention and 388 patients with conventional chemotherapy mentioned about the $\mathrm{CD}_{4}{ }^{+} / \mathrm{CD}_{8}{ }^{+}$. The results showed that the SQI 
intervention group was superior to the control group in improving the $\mathrm{CD}_{4}{ }^{+} / \mathrm{CD}_{8}{ }^{+}$ratio $(\mathrm{RR}=0.27,95 \% \mathrm{CI} 0.21-$ $0.33, P<0.00001)$. Thirteen trials mentioned about $\mathrm{CD}_{8}{ }^{+}$cell levels. However, no statistical significance appeared between 490 patients in the SQI intervention group and 471 patients with conventional chemotherapy. The details concerning the results of the meta-analysis for clinical outcomes in lung cancer patients were illustrated in Figure 2.

\subsection{The Results of Meta-Analysis for Clinical Outcomes in} Digestive Tract Cancer Patients: Objective Tumour Response and Immunity Indicators. Regarding the objective tumour response in digestive tract cancer, there were 11 studies including 397 patients in the SQI intervention group and 403 patients with conventional chemotherapy [37-41, 43, $44,47,54,56]$. The objective tumour response in the SQI intervention group was better than control ( $R R=1.32,95 \%$ CI 1.15-1.52, $P<0.0001)$.

Regarding the NK level variations in digestive tract cancer, there were 6 clinical trials [35, 40, 42, 46, 47, 55] including 204 patients in the SQI intervention group and 194 patients with conventional chemotherapy as control. SQI could significantly improving the NK levels versus control ( $\mathrm{RR}=8.02,95 \% \mathrm{CI} 4.55-11.49, P<0.00001)$. In 10 clinical trials $[36,39,40,43,45-47,50,52,56]$ including 405 patients in the SQI intervention and 376 patients with conventional chemotherapy, the $\mathrm{CD}_{3}{ }^{+}$cell levels in digestive tract cancer were statistically significant improved by SQI $(\mathrm{RR}=9.12,95 \%$ CI 7.00-11.25, $P<0.0001)$. SQI could also improve $\mathrm{CD}_{4}{ }^{+}$cell levels according to 16 trials $[35,36,39,40,42,43,45-47,49-$ $53,55,56]$ including 608 patients in the SQI intervention and 575 patients with conventional chemotherapy $(\mathrm{RR}=7.82$, 95\% CI 6.20-9.43, $P<0.0001)$. The $\mathrm{CD}_{4}{ }^{+} / \mathrm{CD}_{8}{ }^{+}$ratio was improved by SQI in 16 clinical trials [35, 36, 39, 40, 42, 43, 45$47,49-56$ ] which include 684 patients in the SQI intervention and 651 patients with conventional chemotherapy $(R R=0.33$, 95\% CI 0.26-0.41, $P<0.0001$ ). Sixteen trials mentioned about $\mathrm{CD}_{8}{ }^{+}$cell levels. There was no statistical significance between 608 patients in the SQI intervention group and 575 patients with conventional chemotherapy. The results of the meta-analysis for clinical outcomes in digestive tract cancer patients were illustrated in Figure 3.

\subsection{The Results of Meta-Analysis for Clinical Outcomes in} Breast Cancer Patients: Objective Tumour Response and Immunity Indicators. Regarding the objective tumour response in breast cancer, there were 4 trials including 173 patients in the SQI intervention group and 154 patients with conventional chemotherapy as control $[8,57,60,61]$. The objective tumour response in the SQI intervention group was better than control ( $\mathrm{RR}=1.31,95 \%$ CI 1.07-1.60, $P=0.008)$. The NK level was significantly improved by SQI according to 3 clinical trials $[8,59,61]$ which include 143 patients in SQI intervention and 131 patients with conventional chemotherapy $(\mathrm{RR}=6.11,95 \% \mathrm{CI} 3.61-8.61, P<0.00001)$. Regarding the $\mathrm{CD}_{3}{ }^{+}$cell levels, there were 4 clinical trials $[8,59-61]$ including 173 patients in SQI intervention and 161 patients with conventional chemotherapy The results showed that the SQI intervention was superior to the control in improving the $\mathrm{CD}_{3}{ }^{+}$cell levels $(\mathrm{RR}=4.82,95 \%$ CI 2.25-7.38, $P=0.0002)$. The $\mathrm{CD}_{4}{ }^{+}$cell levels was improved by SQI based on 5 clinical trials $[8,58-61]$ including 205 patients in the SQI intervention group and 185 patients with conventional chemotherapy $(\mathrm{RR}=6.58,95 \% \mathrm{CI} 1.60-11.56, P=0.010)$. The $\mathrm{CD}_{4}{ }^{+} / \mathrm{CD}_{8}{ }^{+}$ratio was also improved by SQI from the same 5 clinical trials mentioned above $[8,58-61](R R=0.33$, 95\% CI 0.07-0.59, $P=0.01)$. Meanwhile, the $\mathrm{CD}_{8}{ }^{+}$cell levels were not significantly decreased by SQI $[8,58-61]$. The details were illustrated in Figure 4.

3.6. Evaluation of Publication Bias. Figure 5 showed the funnel plot based on studies with data on the objective tumour response in lung cancer, digestive tract cancer, and breast cancer patients. The funnel plots indicated asymmetry, which might be due to an insufficient number of trials and significant statistical heterogeneity, suggesting that there might be publication bias.

\section{Discussion}

SQI, a formulation injection made from Chinese medical materials through modern preparation technology, is the representative Chinese medicine formula of nourishing vitality and has been used for adjuvant treatment of lung cancer and gastric cancer since being approved by the SFDA in China in 1999. SQI is given by intravenous drip once per day and initiated three days before chemotherapy. SQI is widely used in clinical practice and had excellent performance from market prospects, achieving sales of 268 million in 2010 and generating approximately 1.3 billion in 2014 [64, 65]. Although its specifications declared that the indications were confined to lung cancer and gastric cancer, other types of cancer patients have been given SQI as a combination drug in the clinic. Its extensive application in the palliative care of cancer was benefited from its definite constitution, stable quality control, and accurate efficacy.

The immune system is the frontline of defense against cancer in human and eliminates cancer cells from normal tissues. Nevertheless, chemotherapy could cause normal function damage by the unselective exhaustion of cancer and normal cells. The activation of immune suppressor mechanisms often appears in cancer patients with chemotherapy [66]. Temporary elimination of IL-10 could overcome the immunosuppressive tumour barrier in mice [67]. The therapeutical potential of the PD-1 and PD-L1 pathway, which is important for $\mathrm{T}$ cell regulation in a variety of infectious, autoimmune, and cancer models in mice, was also maximised in recent years. PD-1 knockout mice develop spontaneous autoimmunity [68]. However, the solution for immunosuppression in cancer survivors with chemotherapy remains unsolved but is urgently needed.

The clinical immunoserologic indexes mainly included $\mathrm{NK}, \mathrm{CD}_{3}{ }^{+}, \mathrm{CD}_{4}{ }^{+}$, and $\mathrm{CD}_{8}{ }^{+}$levels and $\mathrm{CD}_{4}{ }^{+} / \mathrm{CD}_{8}{ }^{+}$ratio. The increases of the $\mathrm{NK}, \mathrm{CD}_{3}{ }^{+}, \mathrm{CD}_{4}{ }^{+}$, and $\mathrm{CD}_{4}{ }^{+} / \mathrm{CD}_{8}{ }^{+}$ratio and the decrease of the $\mathrm{CD}_{8}{ }^{+}$level showed improvement of immunosuppressive status. It was demonstrated that SQI interventions showed better performance than conventional chemotherapy treatment in terms of improving immunity 


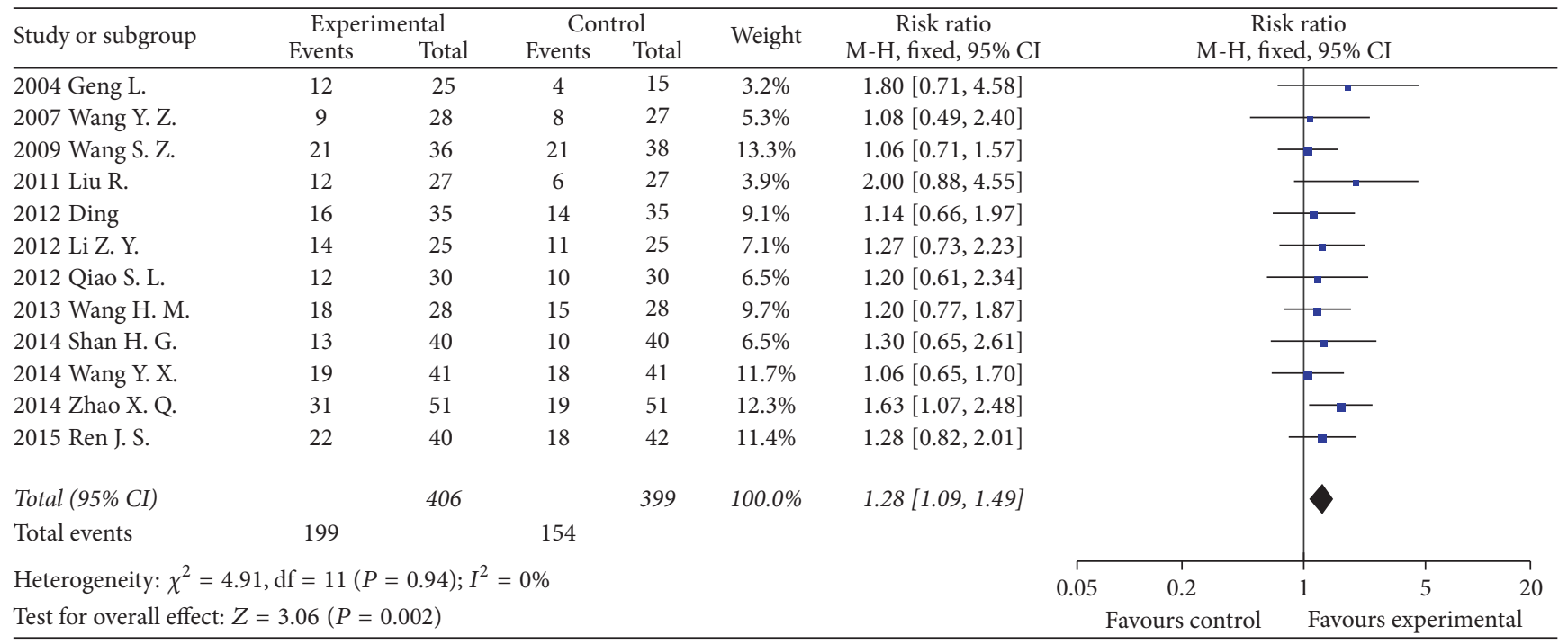

(a)

\begin{tabular}{|c|c|c|c|c|c|c|c|c|c|c|c|}
\hline \multirow{2}{*}{ Study or subgroup } & \multicolumn{3}{|c|}{ Experimental } & \multicolumn{3}{|c|}{ Control } & \multirow{2}{*}{ Weight } & \multirow{2}{*}{$\begin{array}{c}\text { Mean difference } \\
\text { IV, random, 95\% CI }\end{array}$} & \multirow{2}{*}{\multicolumn{3}{|c|}{$\begin{array}{c}\text { Mean difference } \\
\text { IV, random, 95\% CI }\end{array}$}} \\
\hline & Mean & $\mathrm{SD}$ & Total & Mean & $\mathrm{SD}$ & Total & & & & & \\
\hline 2004 Geng L. & 19.2 & 4.1 & 25 & 16 & 3.8 & 15 & $7.4 \%$ & $3.20[0.69,5.71]$ & & -1 & \\
\hline 2005 Jiang Y. & 63.57 & 10.11 & 35 & 49.32 & 8.76 & 32 & $6.3 \%$ & $14.25[9.73,18.77]$ & & & - \\
\hline 2007 Liu L. & 25.22 & 15.1 & 30 & 21.36 & 12.86 & 30 & $4.8 \%$ & $3.86[-3.24,10.96]$ & & & \\
\hline 2007 Sun et al. & 25.82 & 5.15 & 34 & 15.61 & 3.48 & 28 & $7.6 \%$ & $10.21[8.05,12.37]$ & & - & \\
\hline 2007 Wang Y. Z. & 21.25 & 2.78 & 28 & 13.25 & 2.45 & 27 & $7.8 \%$ & $8.00[6.62,9.38]$ & & $\pi$ & \\
\hline 2009 Wang S. Z. & 31.31 & 3.68 & 36 & 19.56 & 2.36 & 38 & $7.8 \%$ & $11.75[10.33,13.17]$ & & - & \\
\hline 2011 Liu Y. F. & 26.37 & 5.34 & 50 & 15.49 & 6.28 & 50 & $7.5 \%$ & $10.88[8.60,13.16]$ & & - & \\
\hline 2012 Ao M. & 24.4 & 2.3 & 30 & 22 & 3.3 & 25 & $7.8 \%$ & $2.40[0.87,3.93]$ & & - & \\
\hline 2012 Ding & 22.4 & 8.37 & 35 & 19.57 & 6.45 & 35 & $6.9 \%$ & $2.83[-0.67,6.33]$ & & - - & \\
\hline 2014 Ren L. & 32.4 & 6.3 & 65 & 14.2 & 5.7 & 72 & $7.6 \%$ & $18.20[16.18,20.22]$ & & & - \\
\hline 2014 Shan H. G. & 26.6 & 2.8 & 40 & 21.5 & 0.8 & 40 & $7.9 \%$ & $5.10[4.20,6.00]$ & & $=$ & \\
\hline 2014 Yuan T. & 22 & 8.8 & 35 & 18.6 & 9.2 & 34 & $6.5 \%$ & $3.40[-0.85,7.65]$ & & & \\
\hline 2014 Zhao X. Q. & 16.96 & 8.65 & 51 & 11.36 & 7.13 & 51 & $7.1 \%$ & $5.60[2.52,8.68]$ & & - & \\
\hline 2015 Ren J. S. & 23.17 & 8.46 & 42 & 17.46 & 7.52 & 42 & $6.9 \%$ & $5.71[2.29,9.13]$ & & - & \\
\hline Total (95\% CI) & & & 536 & & & 519 & $100.0 \%$ & $7.64[5.17,10.11]$ & & & \\
\hline \multicolumn{9}{|c|}{$\begin{array}{l}\text { Heterogeneity: } \tau^{2}=19.78 ; \chi^{2}=260.75, \mathrm{df}=13(P<0.00001) ; I^{2}=95 \% \\
\text { Test for overall effect: } Z=6.07(P<0.00001)\end{array}$} & $\begin{array}{cc}-20 & -10 \\
\text { Favours control }\end{array}$ & $\begin{array}{lc}0 & 10 \\
\text { Favours e }\end{array}$ & 20 \\
\hline
\end{tabular}

(b)

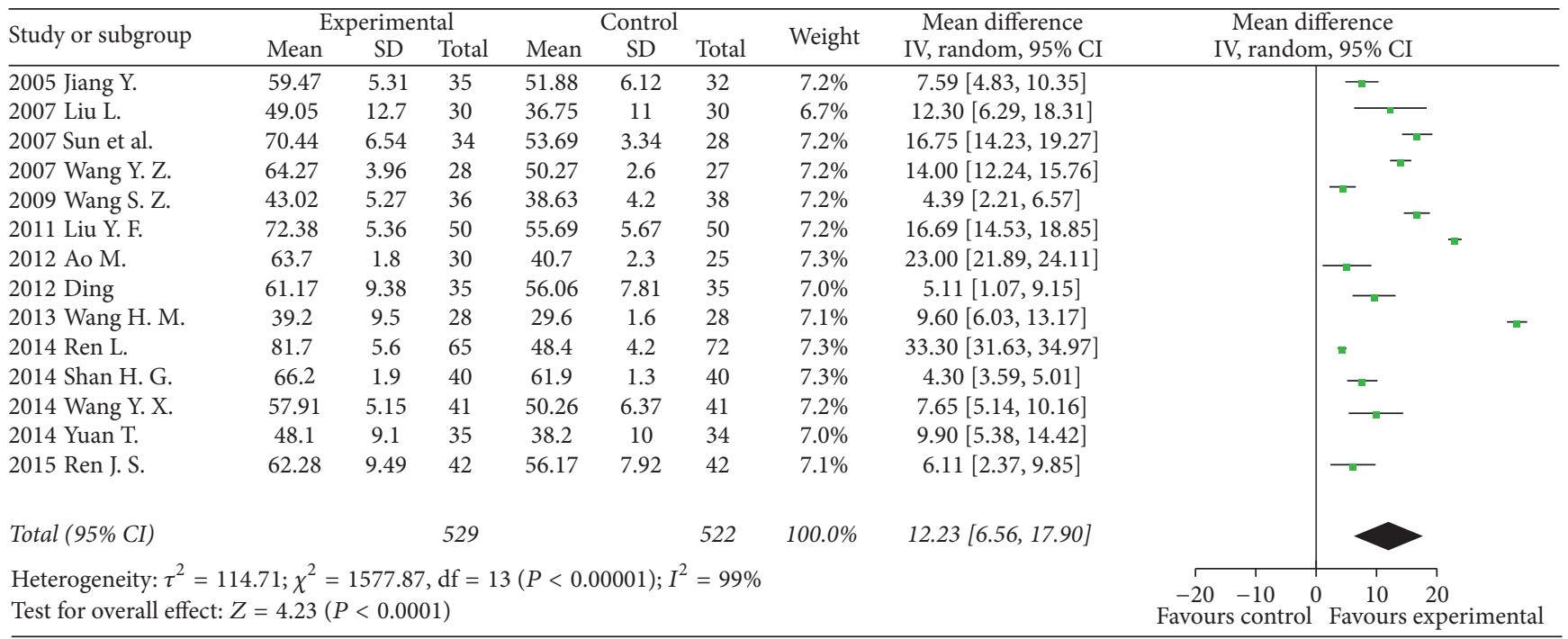

(c)

Figure 2: Continued. 


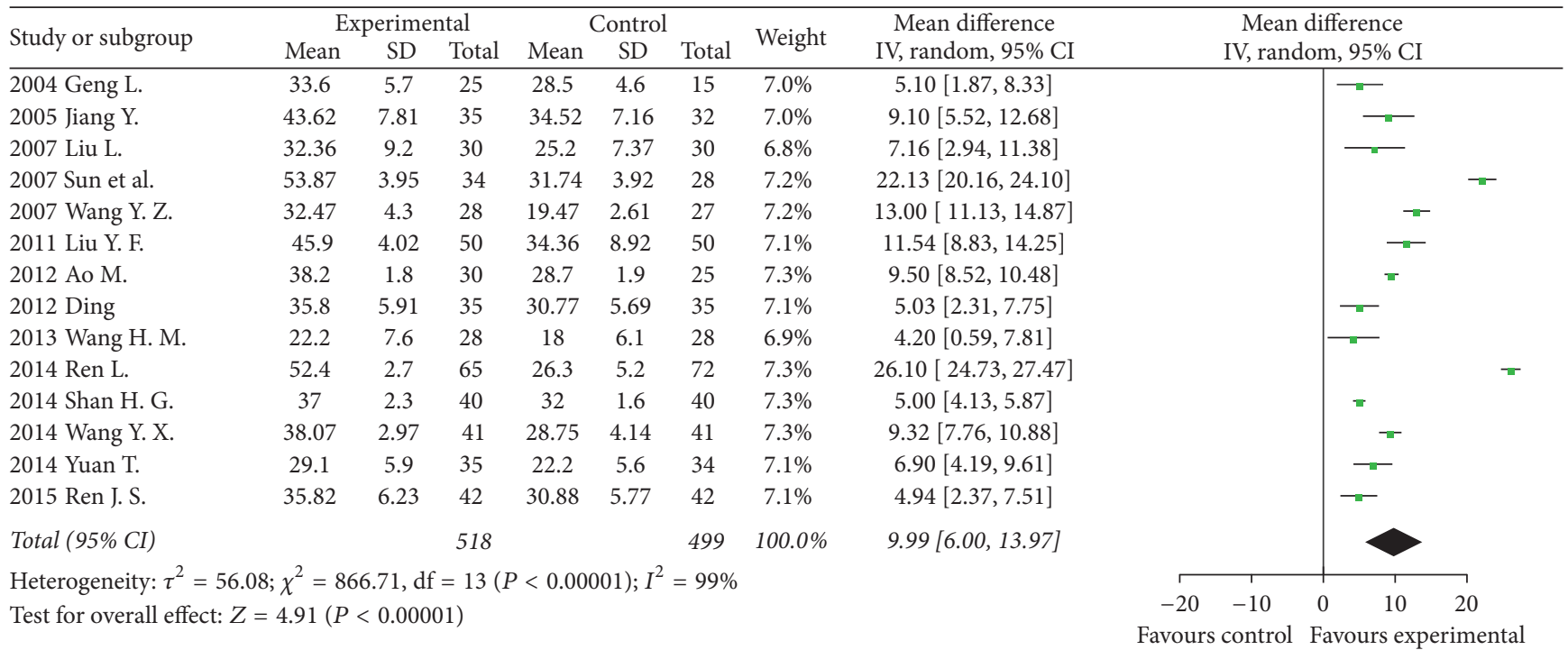

(d)

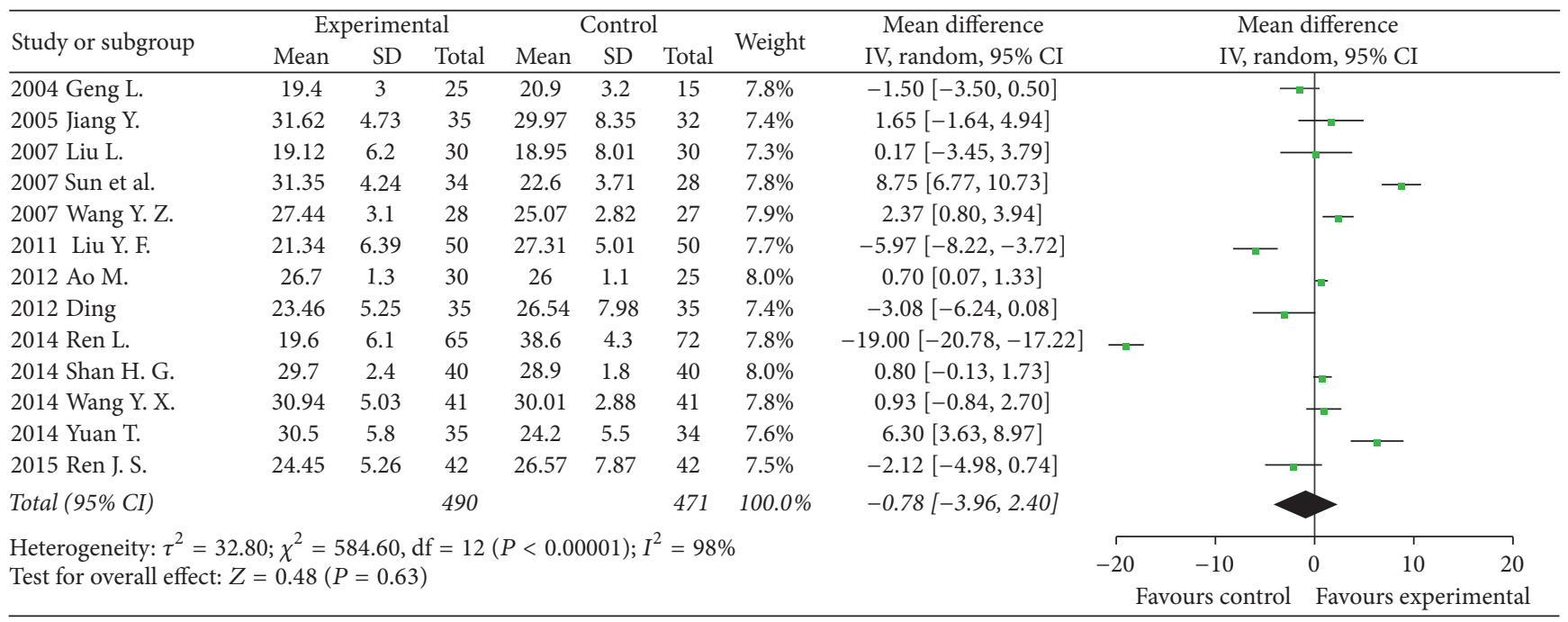

(e)

\begin{tabular}{|c|c|c|c|c|c|c|c|c|c|c|c|}
\hline \multirow{2}{*}{ Study or subgroup } & \multicolumn{3}{|c|}{ Experimental } & \multicolumn{3}{|c|}{ Control } & \multirow{2}{*}{ Weight } & \multirow{2}{*}{$\begin{array}{c}\text { Mean difference } \\
\text { IV, random, 95\% CI }\end{array}$} & \multirow{2}{*}{\multicolumn{3}{|c|}{$\begin{array}{c}\text { Mean difference } \\
\text { IV, random, 95\% CI }\end{array}$}} \\
\hline & Mean & $\mathrm{SD}$ & Total & Mean & $\mathrm{SD}$ & Total & & & & & \\
\hline 2004 Geng L. & 1.58 & 0.53 & 25 & 1.47 & 0.65 & 15 & $2.1 \%$ & $0.11[-0.28,0.50]$ & & & \\
\hline 2005 Jiang Y. & 1.76 & 0.98 & 35 & 0.83 & 0.72 & 32 & $1.9 \%$ & $0.93[0.52,1.34]$ & & & \\
\hline 2007 Liu L. & 1.67 & 0.76 & 30 & 1.33 & 1.02 & 30 & $1.6 \%$ & $0.34[-0.12,0.80]$ & & & \\
\hline 2007 Sun et al. & 1.72 & 0.35 & 34 & 1.4 & 0.21 & 28 & $9.3 \%$ & $0.32[0.18,0.46]$ & & - & \\
\hline 2009 Wang S. Z. & 1.39 & 0.14 & 36 & 1.24 & 0.19 & 38 & $14.9 \%$ & $0.15[0.07,0.23]$ & & -- & \\
\hline 2012 Ao M. & 1.4 & 0.2 & 30 & 1.1 & 0.2 & 25 & $12.1 \%$ & $0.30[0.19,0.41]$ & & $-\square$ & \\
\hline 2012 Ding & 1.57 & 0.34 & 35 & 1.28 & 0.46 & 35 & $6.5 \%$ & $0.29[0.10,0.48]$ & & 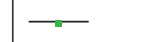 & \\
\hline 2013 Wang H. M. & 1 & 0.4 & 28 & 0.8 & 0.3 & 28 & $6.7 \%$ & $0.20[0.01,0.39]$ & & 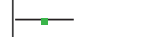 & \\
\hline 2014 Shan H. G. & 1.3 & 0.1 & 40 & 1 & 0.1 & 40 & $17.9 \%$ & $0.30[0.26,0.34]$ & & $=$ & \\
\hline 2014 Wang Y. X. & 1.22 & 0.24 & 41 & 0.94 & 0.29 & 41 & $11.3 \%$ & $0.28[0.16,0.40]$ & & - & \\
\hline 2014 Yuan T. & 0.9 & 0.3 & 35 & 0.7 & 0.4 & 34 & $7.7 \%$ & $0.20[0.03,0.37]$ & & - & \\
\hline 2015 Ren J. S. & 1.54 & 0.31 & 42 & 1.25 & 0.43 & 42 & $8.1 \%$ & $0.29[0.13,0.45]$ & & - & \\
\hline Total (95\% CI) & & & 411 & & & 388 & $100.0 \%$ & $0.27[0.21,0.33]$ & & 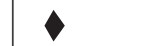 & \\
\hline \multicolumn{9}{|c|}{$\begin{array}{l}\text { Heterogeneity: } \tau^{2}=0.00 ; \chi^{2}=24.30, \mathrm{df}=11(P=0.01) ; I^{2}=55 \% \\
\text { Test for overall effect: } Z=8.95(P<0.00001)\end{array}$} & \begin{tabular}{cc} 
& \multicolumn{1}{c}{1} \\
-1 & -0.5 \\
Favours control
\end{tabular} & $\begin{array}{ll}0 & 0.5 \\
\text { Favours expe }\end{array}$ & $\begin{array}{l}1 \\
\text { erimental }\end{array}$ \\
\hline
\end{tabular}

(f)

FIGURE 2: Forest plots of studies comparing Shenqi Fuzheng injection (SQI) invention groups and control groups, measuring the effect of

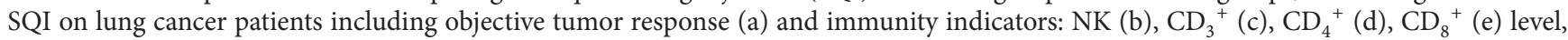
and $\mathrm{CD}_{4}{ }^{+} / \mathrm{CD}_{8}{ }^{+}$ratio (f). 


\begin{tabular}{|c|c|c|c|c|c|c|c|c|c|c|}
\hline \multirow{3}{*}{$\begin{array}{l}\text { Study or subgroup } \\
2002 \text { Sun et al. }\end{array}$} & \multicolumn{2}{|c|}{ Experimental } & \multicolumn{2}{|c|}{ Control } & \multirow{3}{*}{$\begin{array}{l}\text { Weight } \\
6.9 \%\end{array}$} & \multirow{3}{*}{$\begin{array}{c}\text { Risk ratio } \\
\text { M-H, fixed, 95\% CI } \\
1.83[1.03,3.25]\end{array}$} & \multirow{2}{*}{\multicolumn{4}{|c|}{$\begin{array}{c}\text { Risk ratio } \\
\text { M-H, fixed, 95\% CI }\end{array}$}} \\
\hline & \multirow{2}{*}{$\begin{array}{c}\text { Events } \\
22\end{array}$} & \multirow{2}{*}{$\begin{array}{c}\text { Total } \\
46\end{array}$} & \multirow{2}{*}{$\begin{array}{c}\text { Events } \\
12\end{array}$} & \multirow{2}{*}{$\begin{array}{c}\text { Total } \\
46\end{array}$} & & & & & & \\
\hline & & & & & & & & & T & \\
\hline 2009 Liang et al. & 48 & 76 & 35 & 76 & $20.3 \%$ & $1.37[1.02,1.85]$ & & & $\longrightarrow-$ & \\
\hline 2011 Guo et al. & 11 & 24 & 11 & 30 & $5.7 \%$ & $1.25[0.66,2.37]$ & & & & \\
\hline 2012 Ren & 15 & 33 & 14 & 32 & $8.2 \%$ & $1.04[0.60,1.79]$ & & & & \\
\hline 2013 Jin D. X. & 29 & 40 & 19 & 40 & $11.0 \%$ & $1.53[1.05,2.23]$ & & & 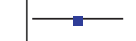 & \\
\hline 2013 Wang W. H. & 21 & 38 & 12 & 38 & $6.9 \%$ & $1.75[1.01,3.03]$ & & & - & \\
\hline 2013 Yin et al. & 14 & 26 & 14 & 27 & $8.0 \%$ & $1.04[0.62,1.73]$ & & & & \\
\hline 2014 Han L. C. & 28 & 34 & 20 & 34 & $11.6 \%$ & $1.40[1.02,1.93]$ & & & & \\
\hline 2014 Wang P. & 18 & 42 & 19 & 42 & $11.0 \%$ & $0.95[0.58,1.53]$ & & & & \\
\hline 2014 Wen J. & 9 & 15 & 8 & 15 & $4.6 \%$ & $1.13[0.60,2.11]$ & & & & \\
\hline 2014 Wen K. & 12 & 23 & 10 & 23 & $5.8 \%$ & $1.20[0.65,2.21]$ & & & & \\
\hline Total (95\% CI) & & 397 & & 403 & $100.0 \%$ & $1.32[1.15,1.52]$ & & & & \\
\hline Total events & 227 & & 174 & & & & & & & \\
\hline \multicolumn{7}{|c|}{ Heterogeneity: $\chi^{2}=6.83, \mathrm{df}=10(P=0.74) ; I^{2}=0 \%$} & 0.2 & 0.5 & 1 & 5 \\
\hline \multicolumn{7}{|c|}{ Test for overall effect: $Z=3.93(P<0.0001)$} & Fav & cont & Favours e & imental \\
\hline
\end{tabular}

(a)

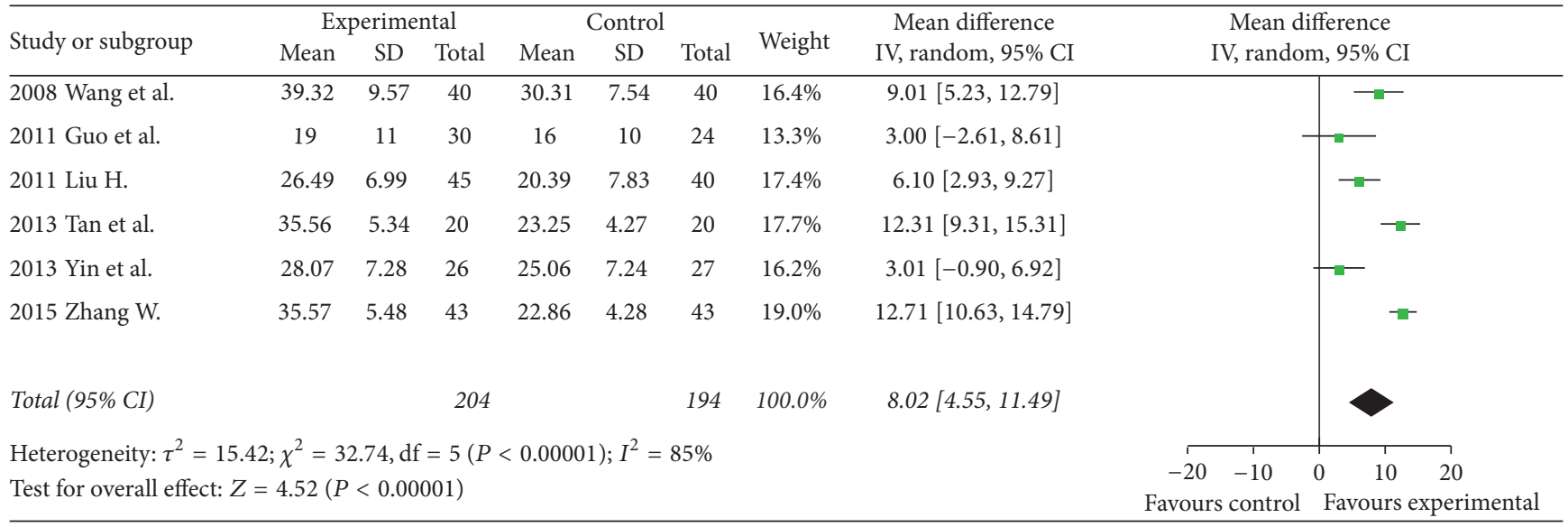

(b)

\begin{tabular}{|c|c|c|c|c|c|c|c|c|c|c|c|c|}
\hline \multirow{2}{*}{ Study or subgroup } & \multicolumn{3}{|c|}{ Experimental } & \multicolumn{3}{|c|}{ Control } & \multirow{2}{*}{ Weight } & \multirow{2}{*}{$\begin{array}{c}\text { Mean difference } \\
\text { IV, random, 95\% CI }\end{array}$} & \multirow{2}{*}{\multicolumn{4}{|c|}{$\begin{array}{c}\text { Mean difference } \\
\text { IV, random, 95\% CI }\end{array}$}} \\
\hline & Mean & SD & Total & Mean & $\mathrm{SD}$ & Total & & & & & & \\
\hline 2002 Sun et al. & 58.76 & 5.82 & 46 & 52.76 & 6.82 & 32 & $13.6 \%$ & $6.00[3.10,8.90]$ & & & $-\square$ & \\
\hline $2009 \mathrm{Ni}$ et al. & 62.05 & 12.05 & 70 & 50.22 & 8.98 & 65 & $12.0 \%$ & $11.83[8.26,15.40]$ & & & $\longrightarrow$ & \\
\hline 2010 Wang W. & 62.46 & 11.97 & 30 & 51.63 & 8.75 & 30 & $8.5 \%$ & $10.83[5.52,16.14]$ & & & & \\
\hline 2011 Guo et al. & 77 & 16 & 30 & 64 & 15 & 24 & $4.8 \%$ & $13.00[4.71,21.29]$ & & & & \\
\hline 2011 Liu H. & 62.57 & 8.43 & 45 & 48.52 & 6.92 & 40 & $12.7 \%$ & $14.05[10.78,17.32]$ & & & & \\
\hline 2012 Chen H. J. & 64.77 & 12.31 & 28 & 61.23 & 11.69 & 28 & $7.0 \%$ & $3.54[-2.75,9.83]$ & & & & \\
\hline 2013 Jin D. X. & 65.2 & 14.6 & 40 & 59.8 & 10.6 & 40 & $8.0 \%$ & $5.40[-0.19,10.99]$ & & & & \\
\hline 2013 Yin L. L. & 64.09 & 7.59 & 26 & 54.64 & 6.39 & 27 & $11.5 \%$ & $9.45[5.67,13.23]$ & & & - & \\
\hline 2014 Han L. C. & 64.78 & 7.88 & 34 & 56.32 & 8.12 & 34 & $11.5 \%$ & $8.46[4.66,12.26]$ & & & -1 & \\
\hline 2014 Yan et al. & 62.06 & 12.54 & 56 & 54.18 & 10.63 & 56 & $10.4 \%$ & $7.88[3.57,12.19]$ & & & - & \\
\hline Total (95\% CI) & & & 405 & & & 376 & $100.0 \%$ & $9.12[7.00,11.25]$ & & & & \\
\hline \multicolumn{9}{|c|}{ Heterogeneity: $\tau^{2}=6.47 ; \chi^{2}=21.81, \mathrm{df}=9(P=0.010) ; I^{2}=59 \%$} & -20 & -10 & 10 & 20 \\
\hline \multicolumn{9}{|c|}{ Test for overall effect: $Z=8.42(P<0.00001)$} & \multicolumn{4}{|c|}{ Favours control Favours experimental } \\
\hline
\end{tabular}

(c)

FIgure 3: Continued. 


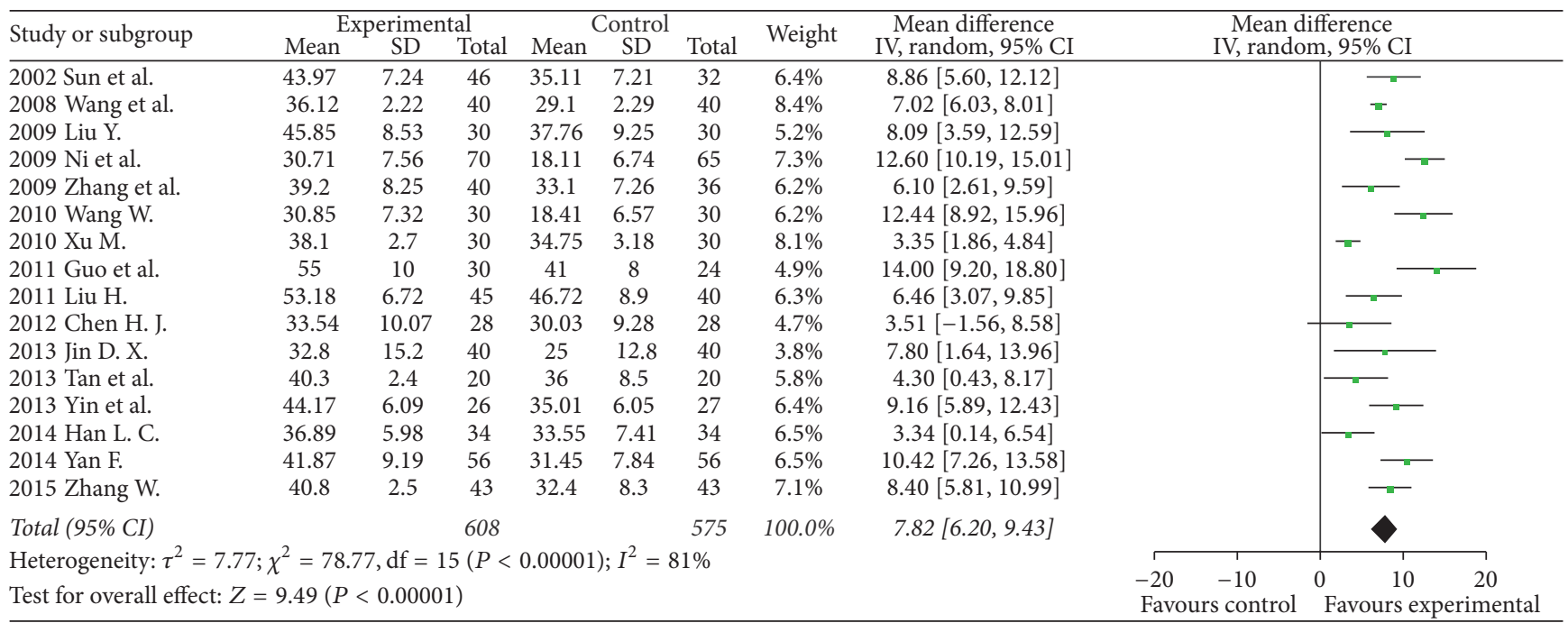

(d)

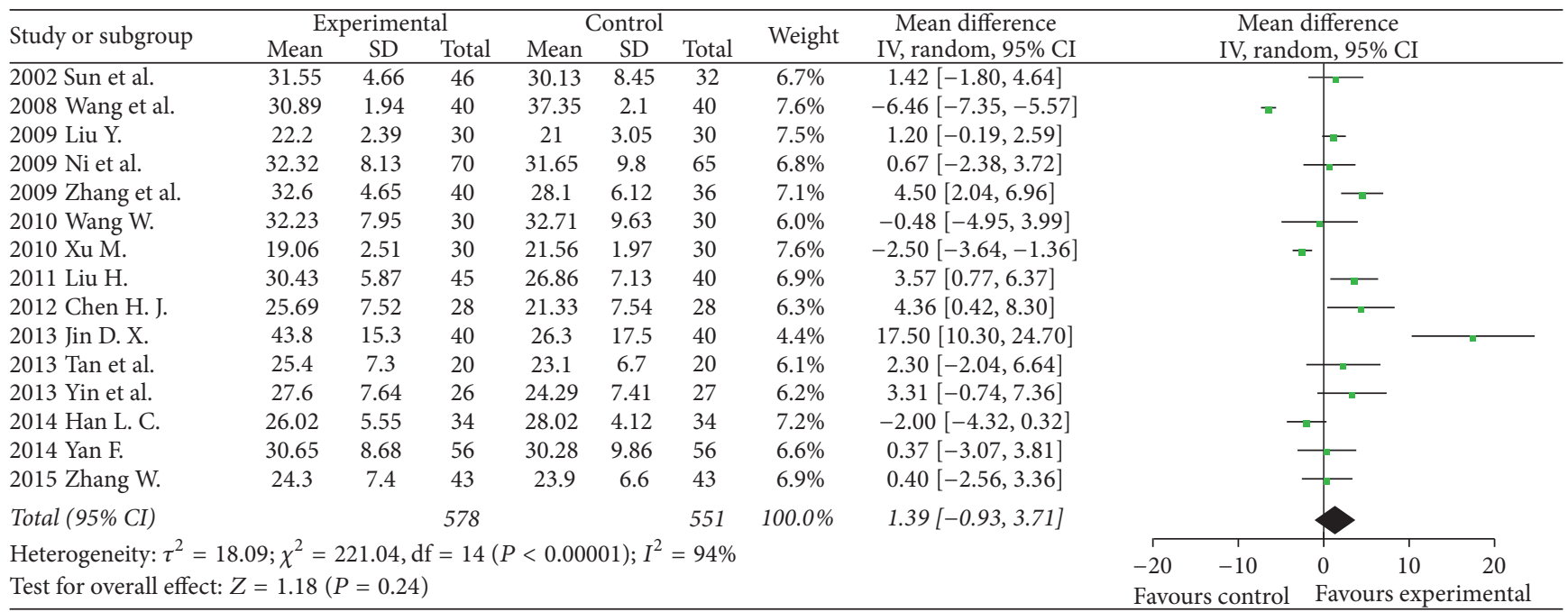

(e)

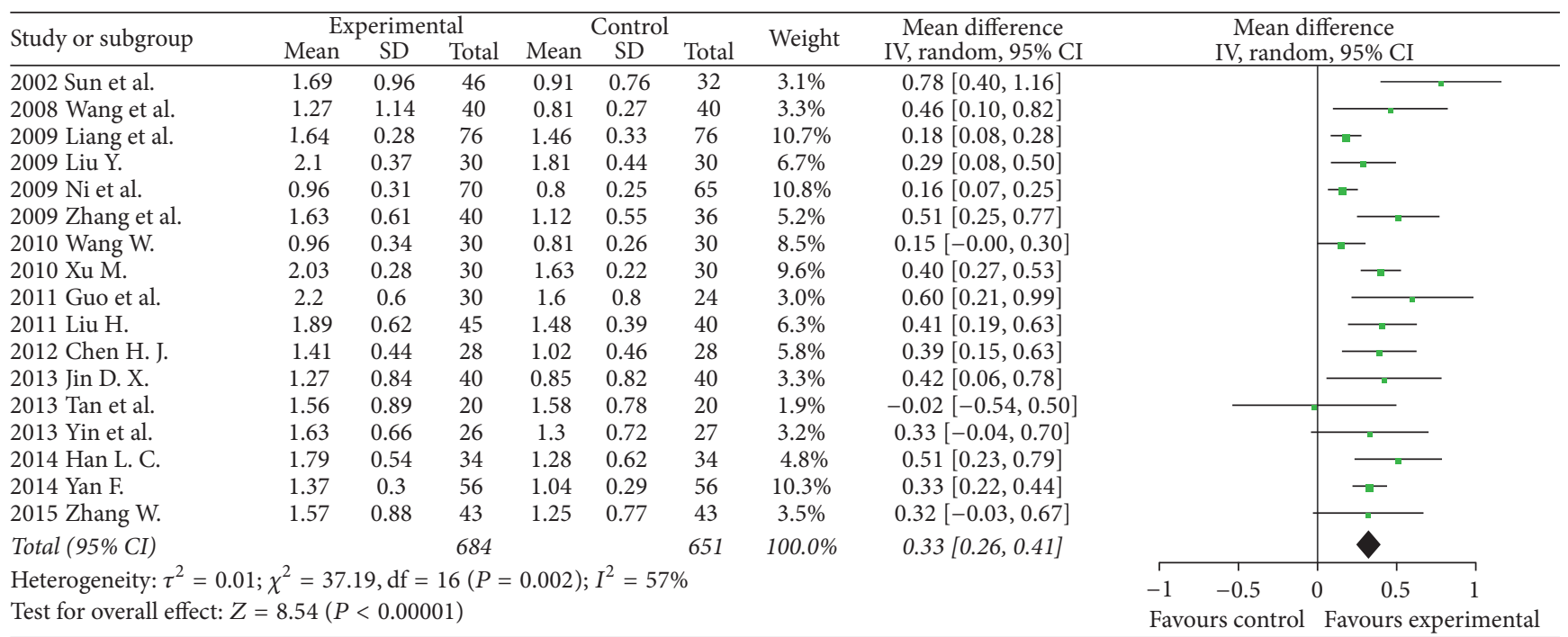

(f)

FIGURE 3: Forest plots of studies comparing Shenqi Fuzheng injection (SQI) invention groups and control groups, measuring the effect of SQI on digestive tract cancer patients including objective tumor response (a) and immunity indicators: $\mathrm{NK}^{(\mathrm{b})}, \mathrm{CD}_{3}{ }^{+}(\mathrm{c}), \mathrm{CD}_{4}{ }^{+}(\mathrm{d}), \mathrm{CD}_{8}{ }^{+}$ (e) level, and $\mathrm{CD}_{4}{ }^{+} / \mathrm{CD}_{8}{ }^{+}$ratio (f). 


\begin{tabular}{|c|c|c|c|c|c|c|c|c|c|c|}
\hline \multirow{3}{*}{$\frac{\text { Study or subgroup }}{2004 \mathrm{Li} \text { and } \mathrm{Ma}}$} & \multicolumn{2}{|c|}{ Experimental } & \multicolumn{2}{|c|}{ Control } & \multirow{2}{*}{ Weight } & \multirow{2}{*}{$\begin{array}{c}\text { Risk ratio } \\
\text { M-H, fixed, 95\% CI }\end{array}$} & \multirow{2}{*}{\multicolumn{4}{|c|}{$\begin{array}{c}\text { Risk ratio } \\
\text { M-H, fixed, 95\% CI }\end{array}$}} \\
\hline & \multirow{2}{*}{$\begin{array}{c}\text { Events } \\
29\end{array}$} & \multirow{2}{*}{$\begin{array}{c}\text { Total } \\
40\end{array}$} & \multirow{2}{*}{$\begin{array}{c}\text { Events } \\
16\end{array}$} & \multirow{2}{*}{$\begin{array}{c}\text { Total } \\
35\end{array}$} & & & & & & \\
\hline & & & & & $21.8 \%$ & $1.59[1.05,2.39]$ & & & $\longrightarrow$ & \\
\hline 2008 Dai et al. & 45 & 65 & 30 & 61 & $39.5 \%$ & $1.41[1.04,1.90]$ & & & 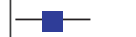 & \\
\hline 2008 Huang et al. & 15 & 30 & 13 & 30 & $16.6 \%$ & $1.15[0.67,1.99]$ & & & - & \\
\hline 2013 Wang D. J. & 20 & 38 & 15 & 28 & $22.1 \%$ & $0.98[0.62,1.55]$ & & & & \\
\hline Total (95\% CI) & & 173 & & 154 & $100.0 \%$ & $1.31[1.07,1.60]$ & & & & \\
\hline Total events & 109 & & 74 & & & & & & & \\
\hline Heterogeneity: $\tau^{2}=$ & $=3(P=$ & 43); $I^{2}$ & $0 \%$ & & & & 0.2 & 0.5 & $1 \quad 2$ & 5 \\
\hline Test for overall effec & $66(P=$ & 08) & & & & & Fav & contr & Favours ex & mental \\
\hline
\end{tabular}

(a)

\begin{tabular}{lccccccccc}
\hline \multirow{2}{*}{ Study or subgroup } & \multicolumn{3}{c}{ Experimental } & \multicolumn{3}{c}{ Control } & \multirow{2}{*}{ Weight } & \multicolumn{2}{c}{ Mean difference } \\
IV, random, 95\% CI
\end{tabular}

(b)

\begin{tabular}{|c|c|c|c|c|c|c|c|c|c|c|}
\hline \multirow{2}{*}{ Study or subgroup } & \multicolumn{3}{|c|}{ Experimental } & \multicolumn{3}{|c|}{ Control } & \multirow{2}{*}{ Weight } & \multirow{2}{*}{$\begin{array}{c}\text { Mean difference } \\
\text { IV, random, 95\% CI }\end{array}$} & \multirow{2}{*}{\multicolumn{2}{|c|}{$\begin{array}{c}\text { Mean difference } \\
\text { IV, random, 95\% CI }\end{array}$}} \\
\hline & Mean & $\mathrm{SD}$ & Total & Mean & SD & Total & & & & \\
\hline $2004 \mathrm{Li}$ and $\mathrm{Ma}$ & 53.9 & 9.6 & 40 & 52.4 & 10.9 & 35 & $18.6 \%$ & $1.50[-3.18,6.18]$ & $\longrightarrow$ & 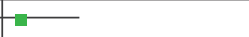 \\
\hline 2008 Dai et al. & 54.11 & 4.31 & 65 & 47.15 & 5.22 & 61 & $40.4 \%$ & $6.96[5.28,8.64]$ & & \\
\hline 2008 Huang et al. & 65.57 & 9.35 & 30 & 62.15 & 8.64 & 30 & $19.2 \%$ & $3.42[-1.14,7.98]$ & & $\rightarrow-$ \\
\hline 2008 Yuan et al. & 50.74 & 9.12 & 38 & 45.84 & 8.67 & 35 & $21.8 \%$ & $4.90[0.82,8.98]$ & & \\
\hline Total (95\% CI) & & & 173 & & & 161 & $100.0 \%$ & $4.82[2.25,7.38]$ & & \\
\hline \multicolumn{9}{|c|}{$\begin{array}{l}\text { Heterogeneity: } \tau^{2}=3.50 ; \chi^{2}=6.30, \mathrm{df}=3(P=0.10) ; I^{2}=52 \% \\
\text { Test for overall effect: } Z=3.68(P=0.0002)\end{array}$} & $\begin{array}{cc}-10 & -5 \\
\text { Favours control }\end{array}$ & $\begin{array}{lll} & 1 & \\
0 & 5 & 10 \\
\text { Favours experimental }\end{array}$ \\
\hline
\end{tabular}

(c)

\begin{tabular}{|c|c|c|c|c|c|c|c|c|c|c|}
\hline \multirow{2}{*}{ Study or subgroup } & \multicolumn{3}{|c|}{ Experimental } & \multicolumn{3}{|c|}{ Control } & \multirow{2}{*}{ Weight } & \multirow{2}{*}{$\begin{array}{c}\text { Mean difference } \\
\text { IV, random, 95\% CI }\end{array}$} & \multirow{2}{*}{\multicolumn{2}{|c|}{$\begin{array}{c}\text { Mean difference } \\
\text { IV, random, 95\% CI }\end{array}$}} \\
\hline & Mean & $\mathrm{SD}$ & Total & Mean & $\mathrm{SD}$ & Total & & & & \\
\hline $2004 \mathrm{Li}$ and $\mathrm{Ma}$ & 38 & 8 & 40 & 35 & 7.3 & 35 & $19.5 \%$ & $3.00[-0.46,6.46]$ & & $F-$ \\
\hline 2008 Dai et al. & 40.26 & 5.15 & 65 & 33.84 & 4.53 & 61 & $21.0 \%$ & $6.42[4.73,8.11]$ & & - \\
\hline 2008 Huang et al. & 39.97 & 6.25 & 30 & 24.19 & 1.23 & 30 & $20.6 \%$ & $15.78[13.50,18.06]$ & & - \\
\hline 2008 Yuan et al. & 37.77 & 7.38 & 38 & 34.27 & 7.12 & 35 & $19.7 \%$ & $3.50[0.17,6.83]$ & & F- \\
\hline 2008 Zhu et al. & 38.79 & 7.26 & 32 & 35.13 & 7.21 & 24 & $19.1 \%$ & $3.66[-0.17,7.49]$ & & $F-$ \\
\hline Total (95\% CI) & & & 205 & & & 185 & $100.0 \%$ & $6.58[1.60,11.56]$ & & \\
\hline \multicolumn{9}{|c|}{ Heterogeneity: $\tau^{2}=29.95 ; \chi^{2}=67.93, \mathrm{df}=4(P<0.00001) ; I^{2}=94 \%$} & $\begin{array}{cc}-20 & -10 \\
\text { Favours control }\end{array}$ & 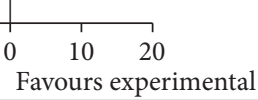 \\
\hline
\end{tabular}

(d)

\begin{tabular}{lcccccccc}
\hline Study or subgroup & \multicolumn{3}{c}{ Experimental } & \multicolumn{3}{c}{ Control } & Weight & \multicolumn{2}{c}{ Mean difference } \\
IV, random, 95\% CI
\end{tabular}

(e)

Figure 4: Continued. 


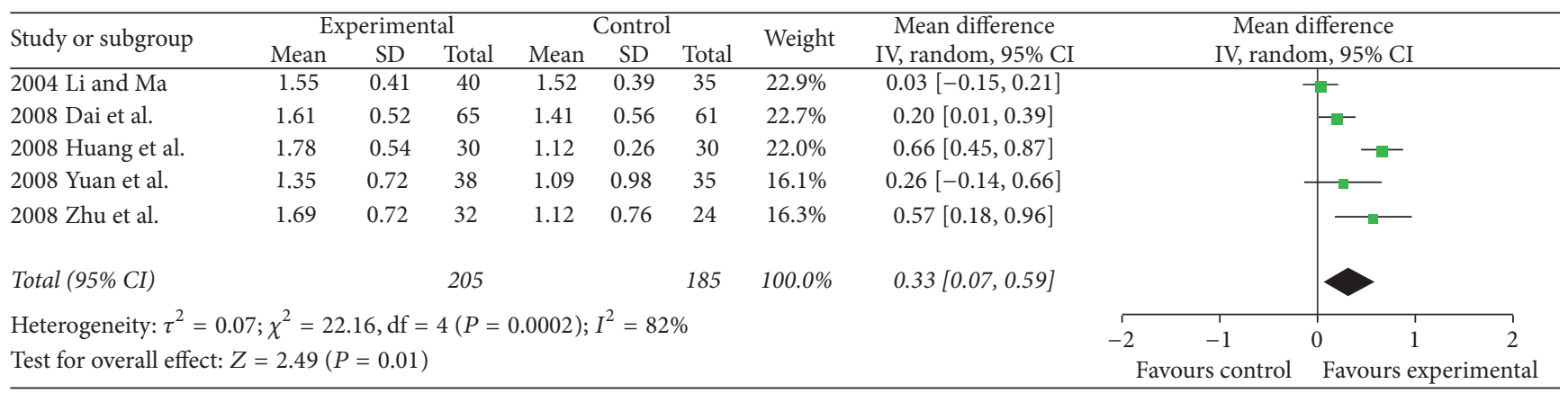

(f)

FIGURE 4: Forest plots of studies comparing Shenqi Fuzheng injection (SQI) invention groups and control groups, measuring the effect of SQI on breast cancer patients including objective tumor response (a) and immunity indicators: $\mathrm{NK}(\mathrm{b}), \mathrm{CD}_{3}^{+}(\mathrm{c}), \mathrm{CD}_{4}^{+}(\mathrm{d}), \mathrm{CD}_{8}{ }^{+}(\mathrm{e})$ level, and $\mathrm{CD}_{4}^{+} / \mathrm{CD}_{8}^{+}$ratio (f).

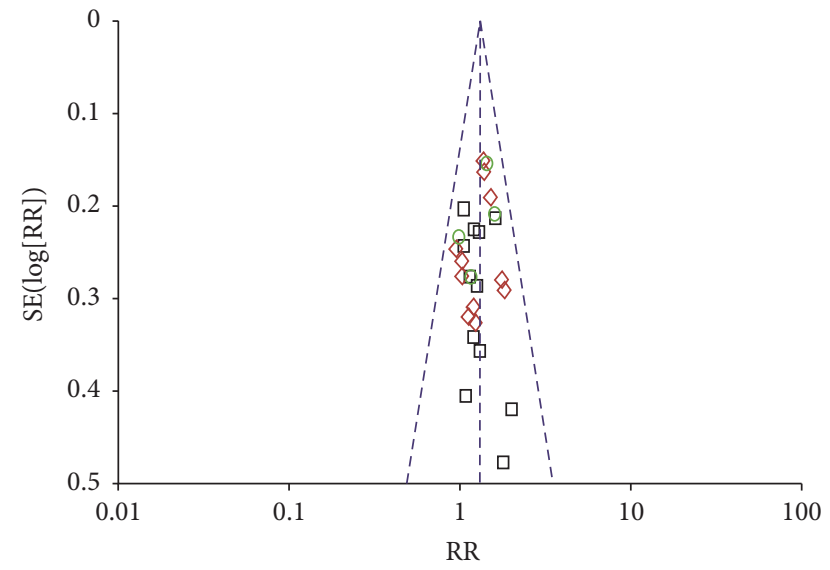

$$
\begin{aligned}
& \text { Subgroups } \\
& \square \text { Lung cancer } \\
& \diamond \text { Digestive tract cancer }
\end{aligned}
$$

FIGURE 5: The funnel plot analysis of publication bias on objective tumor response data of lung cancer, digestive tract cancer, and breast cancer patients.

parameters with enhanced $\mathrm{NK}, \mathrm{CD}_{3}{ }^{+}$, and $\mathrm{CD}_{4}{ }^{+}$levels and $\mathrm{CD}_{4}{ }^{+} / \mathrm{CD}_{8}{ }^{+}$ratio, suggesting that SQI had a good effect on immune system damage caused by chemotherapy.

Nevertheless, all studies included in the analysis were of low quality according to the Jadad scale. A random allocation was mentioned in all Chinese-language articles; however, the detailed methods of allocation concealment were not described in any articles, which might have led to selection bias and overestimation of the intervention effects. Furthermore, the included trials lacked follow-up outcome indicators to determine the long-term curative effect. The majority of the included trials were classified into three categories: lung cancer, digestive tract cancer, and breast cancer. There were also studies scattered in other cancers such as leukemia and cervical cancer [69-71]. However, those trials were insufficient for conducting a meta-analysis. The metaanalysis of this paper showed comparatively higher heterogeneity for immunity indicators, which might be because the studies included measured different treatment effects under various cancers instead of measuring a single disease effect.

\section{Conclusions}

Although SQI intervention showed immunity enhancement in chemotherapy cancer patients statistically, the metaanalysis results in this paper should be prudently adopted in clinical practice. Although placebo-controlled and doubleblinded clinical trials of sizeable samples regarding SQI interventions should be conducted, this meta-analysis still provides useful information for clinical practice.

\section{Competing Interests}

The authors declare that there is no conflict of interests regarding the publication of this paper.

\section{Authors' Contributions}

Yang Yang and Wang Ting contributed equally.

\section{Acknowledgments}

This work was supported by the National Program for Key Basic Research Projects (2012CB518404) and the Ministry of Education of PRC "Program for Innovative Research Team in University” (no. IRT1276).

\section{References}

[1] J. R. Carver, "Cardiac toxicity in the elderly and the role of cardio oncology," Progress in Pediatric Cardiology, vol. 39, no. 2, pp. 7175, 2015.

[2] M. Farooqui, M. A. Hassali, A. K. Abdul Shatar et al., "Complementary and alternative medicines (CAM) disclosure to the health care providers: a qualitative insight from Malaysian cancer patients," Complementary Therapies in Clinical Practice, vol. 18, no. 4, pp. 252-256, 2012.

[3] L. Li and P. S. Leung, "Use of herbal medicines and natural products: an alternative approach to overcoming the apoptotic 
resistance of pancreatic cancer," International Journal of Biochemistry and Cell Biology, vol. 53, pp. 224-236, 2014.

[4] B. Poonthananiwatkul, R. L. Howard, E. M. Williamson, and R. H. M. Lim, "Cancer patients taking herbal medicines: a review of clinical purposes, associated factors, and perceptions of benefit or harm," Journal of Ethnopharmacology, vol. 175, pp. 58-66, 2015.

[5] B. Patwardhan, "Traditional medicine-inspired evidence-based approaches to drug discovery," in Evidence-Based Validation of Herbal Medicine, P. K. Mukherjee, Ed., chapter 12, pp. 259-272, Elsevier, Boston, Mass, USA, 2015.

[6] Q. Ai, W. Zhang, Y. Xie, W. Huang, H. Liang, and H. Cao, "Post-marketing safety monitoring of Shenqifuzheng injection: a solution made of Dangshen (Radix Codonopsis) and Huangqi (Radix Astragali Mongolici)," Journal of Traditional Chinese Medicine, vol. 34, no. 4, pp. 498-503, 2014.

[7] J. Wang, X. Tong, P. Li, H. Cao, and W. Su, "Immunoenhancement effects of Shenqi Fuzheng Injection on cyclophosphamide-induced immunosuppression in Balb/c mice," Journal of Ethnopharmacology, vol. 139, no. 3, pp. 788-795, 2012.

[8] Z. Dai, X. Wang, H. Kang et al., "Clinical effects of Shenqi Fuzheng injection in the neoadjuvant chemotherapy for local advanced breast cancer and the effects on T-lymphocyte subsets," Journal of Traditional Chinese Medicine, vol. 28, no. 1, pp. 34-38, 2008.

[9] M. Li, C. Qiao, L. Qin, J. Zhang, and C. Ling, "Application of Traditional Chinese Medicine injection in treatment of primary liver cancer: a review," Journal of Traditional Chinese Medicine, vol. 32, no. 3, pp. 299-307, 2012.

[10] J. Wang, X. Tong, P. Li et al., "Bioactive components on immuno-enhancement effects in the traditional Chinese medicine Shenqi Fuzheng Injection based on relevance analysis between chemical HPLC fingerprints and in vivo biological effects," Journal of Ethnopharmacology, vol. 155, no. 1, pp. 405415, 2014.

[11] L. Askie and M. Offringa, Systematic Reviews and MetaAnalysis, Seminars in Fetal and Neonatal Medicine, 2010.

[12] R. Sylvester, "WHO handbook for reporting results of cancer treatment: WHO offset publication \#48 World Health Organization, Geneva, 1979, 45 pages, 6 Swiss Francs," Controlled Clinical Trials, vol. 1, no. 3, pp. 276-277, 1980.

[13] H. D. Clark, G. A. Wells, C. Huët et al., "Assessing the quality of randomized trials: reliability of the Jadad scale," Controlled Clinical Trials, vol. 20, no. 5, pp. 448-452, 1999.

[14] T. Le, J. E. Hornyak, and D. Kim, "Poster 354 assessment of randomized controlled studies in physical medicine and rehabilitation using the jadad scale: a systematic review," $P M \ll R$, vol. 4, no. 10, supplement, p. S311, 2012.

[15] D. V. Lindley, "Hierarchical models: random and fixed effects," in International Encyclopedia of the Social Sciences, J. D. Wright, Ed., pp. 836-840, Elsevier, Oxford, UK, 2015.

[16] J. S. Ren, "Preventive effect of shenqi fuzheng injection on adverse reactions of chemotherapy in the treatment of advanced non small cell lung cancer," Modern Journal of Integrated Traditional Chinese and Western Medicine, vol. 24, no. 12, p. 1286, 2015.

[17] X. Q. Zhao, "Clinical research of combined therapy of Chinese and Western medicine on advanced non small cell lung cancer," Hebei Journal of Traditional Chinese Medicine, no. 7, pp. 10291031, 2014.
[18] T. Yuan, "Clinical observation of efficacy on Shenqifuzheng injection coupled with chemotherapy on non small cell lung cancer patients," Henan Medical Research, vol. 23, no. 6, p. 123, 2014.

[19] Y. X. Wang and C. F. Dou, "Clinical observation of efficacy on Shenqifuzheng injection coupled with chemotherapy on 41 cases of non small cell lung cancer patients," Journal of Traditional Chinese Medicine, vol. 55, no. 9, p. 775, 2014.

[20] L. Ren, "Effect of Shenqi Fuzheng Injection on the immunologic and hematopoietic function in patients with advanced nonsmall cell lung cancer treated with chemotherapy," Chinese Journal of Clinical Oncology and Rehabilitation, no. 4, pp. 463465, 2014.

[21] P. J. Q. Shan, H. G. Zhou, and X. F. Zhou, "Effect evaluation on DP regimen combined with Shenqi fuzheng injection in treating 40 cases of non small cell lung cancer," Chinese Pharmaceutical Journal, vol. 23, no. 24, p. 25, 2014.

[22] R. F. Wang, H. M. Lin, and M. X. Lin, "Combination of Shenqi fuzheng injection and EP chemotherapy for small cell lung cancer," Shanghai University of Traditional Chinese Medicine, vol. 47, no. 3, p. 46, 2013.

[23] S. L. Qiao, "Effect of Shenqi Fuzheng injection combined with chemotherapy in treatment of advanced non small cell lung cancer clinical observation," China Practical Medical, vol. 7, no. 34, pp. 25-26, 2012.

[24] Z. Y. Li, "Clinical observation of shenqi fuzhegn injection with chemotherapy for the treatment of 25 cases of non small cell lung cancer," Journal of Baotou Medical, vol. 36, no. 4, p. 226, 2012.

[25] C. J. Ding and L. Yang, "Influence of ShenQi FuZheng injection on immune function of chemotherapeutic patients with advanced non-small cell lung cancer," Western Journal Traditional Chinese Medicine, vol. 25, no. 1, p. 7, 2012.

[26] L. X. R. Ao, M. Liu, C. S. Li, and Q. S. Li, "Effect of Shenqi Fuzheng Injecfion on hematopoietic and immune functions in patients with lung cancer undergoing chemotherapy," Shandong Medical Journal, vol. 52, no. 3, p. 60, 2012.

[27] Y. F. Liu and L. L. Ren, "Effect of Shenqi Fuzheng injection combined with chemotherapy in treating advanced non small cell lung cancer patients," China Journal of Modern Medicine, vol. 18, no. 10, p. 83, 2011.

[28] R. Liu, "Clinical observation of efficacy on Shenqifuzheng injection coupled with chemotherapy on advanced non small cell lung cancer patients," Journal of Medical Theory and Practice, vol. 24, no. 11, p. 1291, 2011.

[29] S. Z. Wang, "Clinical observation of efficacy on Shenqifuzheng injection coupled with chemotherapy on 74 cases of advanced lung cancer patients," Journal of Emergency in Traditional Chinese Medicine, vol. 18, no. 8, p. 1231, 2009.

[30] Y. Z. X. Wang, Y. Z. Liao, and S. H. Shen, "Effect of shenqi fuzheng injection with domestic vinorelbine and carboplatin for the treatment of advanced non-small cell lung cancer," Journal of Clinical Internal Medicine, vol. 24, no. 3, p. 206, 2007.

[31] Y. Y. Sun, S. J. Ma, Y. J. Jia, Y. Zhang, and L. Chen, "Effects of cellular immunity state of body and toxic effect of the treatment by chemotherapy Combmed with Shenqi fuzheng injection on non small cell lung cancer patients," Traditional Chinese Medicine, Emergency Medicine, vol. 16, no. 7, p. 776, 2007.

[32] H. S. Lin and D. R. Li, "Multi-center randomized clinical study on Shenqi-fuzheng injection combined with chemotherapy in the treatment for lung cancer," Chinese Journal Of Oncology, vol. 29, no. 12, pp. 931-934, 2007. 
[33] Y. Jiang and W. B. Zhuang, "Clinical observation of efficacy on Shenqifuzheng injection coupled with chemotherapy on advanced lung caner patients," Oncology Program, vol. 2, no. 10, p. 137, 2004.

[34] G. Li, "Clinical research of Shenqifuzheng Injection combined with chemotherapy to Treat non small cell lung cancer patients," Journal of Medical Forum, vol. 25, no. 17, pp. 29-30, 2004.

[35] W. Zhang, H. Y. Sun, and W. E. Zheng, "Effect of Shenqi Fuzheng injection on life quality and immune function the patients after operation with advance colon cancer," Modern Practical Medicine, vol. 27, no. 3, p. 358, 2015.

[36] F. Yan, Y. M. Zhou, and P. Jian, "Effect of Shenqifuzheng injection combined with chemotherapy on immune function in patients with colon cancer after operation," China Journal of Modern Medicine, vol. 24, no. 16, p. 72, 2014.

[37] J. Wen, Clinical Observation of Advanced Gastric Cancer Treated with Shenqi Fuzheng Injection Combined with Chemotherapy, Yangzhou University, Yangzhou, China, 2014.

[38] P. Wang, "Clinical observation of efficacy and immnity function on Shenqifuzheng injection coupled with chemotherapy on advanced gastric cancer patients," Clinical Medicine, vol. 34, no. 8, p. 124, 2014.

[39] H. N. Han, L. C. Liu, Y. T. Li et al., "Influence of Shenqi Fuzheng injection on immune function of advanced gastric cancer patients in chemotherapy," Liaoning University of Traditional Chinese Medicine, vol. 16, no. 11, 158, 2014.

[40] L. L. Yin and C.-Y. Jiang, "Observation on the influence of Shenqi-Fuzheng injection on T-lymphocyte subsets, NK cell and the leukocyte of the patients with advanced gastric cancer," International Journal of Traditional Chinese Medicine, vol. 35, no. 1, pp. 22-24, 2013.

[41] W. H. Wang, "Clinical efficacy of Shenqi fuzheng injection on patients with advanced gastrointestinal cancer undergoing chemotherapy," Strait Pharmaceutical Journal, vol. 15, no. 1, p. 113, 2013.

[42] G. G. Tan, L. Liu, J. Li, and Y.-Z. Hu, "Effects of Shenqifuzheng injection on immunolologic function of patients with colon cancer," Journal of Hainan Medical University, vol. 19, no. 5, p. 627, 2013.

[43] D. X. Jin, "Clinical value analysis of Shenqi Fuzheng injection chemotherapy for advanced gastric cancer," Practical Pharmacy and Clinical Remedies, vol. 16, no. 5, p. 451, 2013.

[44] Y. Z. Ren and F. Wang, "Influence of Advanced Gastric Cancer Treated with Shenqi Fuzheng Injection combined with chemotherapy on effect and immune function," Journal of Basic and Clinical Oncology, vol. 25, no. 5, p. 394, 2012.

[45] C. Huajun and X. Xinmei, "Effect of Shenqi Fuzheng Injecfion on immune function in patients with advanced malignant gastrointestinal tumors," Journal of Modern Medicine \& Health, no. 16, pp. 2448-2449, 2012.

[46] H. Liu and D. Han, "Effects of Shenqifuzheng injection on the immunolologic function and clinic efficacy in the elderly patients with advanced gastric cancer," Chinese Journal of Clinical Pharmacology, vol. 27, no. 3, p. 177, 2011.

[47] Y. R. Guo, H. P. Zhao, and B. Chai, "Shenqi fuzheng injection with oxaliplatin, 5-fluorouracil and leucovorin calciumfor the treatment of 30 cases of middle-late colorectal cancer patients," Chinese Remedies and Clinics, vol. 11, no. 2, p. 220, 2011.

[48] Y. Zhang, L.-L. Guo, and S.-P. Zhao, "Effect of shenql fuzheng injection combined with chemotherapy in treating colorectaI cancer," Chinese Journal of Integrated Traditional and Western Medicine, vol. 30, no. 3, pp. 280-282, 2010.
[49] M. Xu, "Effect of chemotherapy with Shenqi Fuzheng Injection on the patients after operation with esophageal carcinoma," Liaoning University of Traditional Chinese Medicine, 2010.

[50] W. Wang, "The immuno-enhancement of Shenqi fuzheng injection on patients with gastrointestinal cancer undergoing chemotherapy", Journal of Contemporary Medicine, vol. 16, no. 32, p. 150, 2010.

[51] X. H. Zhang, Y. Z. Li, Y. Q. Wang, and X. Q. Yang, "Effect of shenqi fuzheng injection on the immune function of patients with colon cancer postoperative undergoing chemotherapy," China Practical Medical, vol. 4, no. 13, p. 129, 2009.

[52] B. Q. Ni, Z. H. Zhang, R. X. Chen, and Z. X. Luo, "Effect of Shenqi Fuzheng injection with chemotherapy on the patients after operation with colorectal cancer," Guangxi Medical Journal, vol. 31, no. 5, p. 644, 2009.

[53] Y. Liu and B. Q. Gong, "Application of Shenqi Fuzheng injection on hyperthermic intraperitoneal lavage chemotherapy for gastric cancer," Journal of Practical Oncology, vol. 23, no. 4, p. 339, 2009.

[54] Q. L. Liang, D. C. Pan, and J. R. Xie, "Effect of Shenqi Fuzheng injection combined with chemotherapy in treating advanced colorectal carcinoma," Chinese Journal of Integrated Traditional and Western Medicine, vol. 29, no. 5, pp. 439-441, 2009 (Chinese).

[55] Y. L. Wang, Y. Gao, H. Yin, and L. Z. Wu, "Effects of Shenqi fuzheng injection on the immune function of patients with malignant gastrointestinal tumors," Traditional Chinese Drug Research \& Clinical Pharmacology, vol. 19, no. 6, p. 506, 2008.

[56] D. X. Sun, W. Y. Qiu, and H. C. Shen, "Clinical observation of efficacy on Shenqifuzheng injection coupled with chemotherapy on advanced gastrointestinal tumor patients," Zhejiang Journal of Integrated Traditional and Western Medicine, vol. 12, no. 5 , p. 284, 2002.

[57] D. J. Wang, "Clinical observation of Chinese and Western drugs for the treatment of 76 cases of breast cancer," Medical Recapitulate, vol. 14, pp. 2676-2678, 2013.

[58] K. Zhu, C. X. Dang, and D. M. Chang, "Effects of shenqi fuzheng injection with CEF chemotherapy on the immune function of breast cancer patients," Chinese Journal of Clinical Oncology, vol. 35, no. 14, p. 808, 2008.

[59] J. W. Yuan, G. Q. Kang, X. F. Liu, and S. J. Yang, "Effects of neoadjuvant chemotherapy combined with shenqifuzheng injection on cell immune function for patients with breast cancer," Lishizhen Medicine and Materia Medica Research, vol. 19, no. 5, pp. 1099-1101, 2008.

[60] Z. F. Huang, H. Z. Li, Z. Q. Tan, Z. J. Zhang, and C. Chen, "Clinical observation of efficacy and adverse reactions on Shenqifuzheng injection used in patients with breast cancer receiving neoadjuvant chemotherapy," in Proceedings of the 11th Chinese Integrative Medicine Oncology Coference, 2008, p. 263.

[61] X. L. Li and W. J. Ma, "Clinical observation of efficacy on Shenqifuzheng injection coupled with chemotherapy on advanced breast caner patients," Modern Oncology, vol. 12, no. 6, p. 574, 2004.

[62] L. Lin, Clinical Observation of Shenqifuzheng Injection Combined with Chemotherapy in Treatment of IIIA Non Small Cell Lung Cancer, LiaoNing University of Traditional Chinese Medicine, 2007.

[63] K. Wen, J. Li, and D. Y. Peng, "Clinical observation of Shenqifuzheng injection combined with XELOX regimen in treatment of advanced gastric cancer," Journal of Hubei College Traditional Chinese Medicine, vol. 16, no. 6, pp. 65-67, 2014. 
[64] Y. Lai, J. Lu, X. Chen, H. Hu, and Y. Wang, "Analysis of anticancer traditional Chinese medicine injections based on market performance," World Science and Technology, vol. 14, no. 5, pp. 1958-1962, 2012.

[65] The Lancet Oncology, "Rethinking traditional Chinese medicines for cancer," The Lancet Oncology, vol. 16, no. 15, p. 1439, 2015.

[66] S. J. Haikerwal, J. Hagekyriakou, M. MacManus, O. A. Martin, and N. M. Haynes, "Building immunity to cancer with radiation therapy," Cancer Letters, vol. 368, no. 2, pp. 198-208, 2015.

[67] J. Rossowska, N. Anger, J. Kicielińska et al., "Temporary elimination of IL-10 enhanced the effectiveness of cyclophosphamide and BMDC-based therapy by decrease of the suppressor activity of MDSCs and activation of antitumour immune response," Immunobiology, vol. 220, no. 3, pp. 389-398.

[68] J. M. Chinai, M. Janakiram, F. Chen, W. Chen, M. Kaplan, and $\mathrm{X}$. Zang, "New immunotherapies targeting the PD-1 pathway," Trends in Pharmacological Sciences, vol. 36, no. 9, pp. 587-595, 2015.

[69] H. B. Cheng and M. Z. Luo, "The clinical observation of shenqi fuzheng injection combined with chemotherapy for the treatment of leukemia," Journal of Oncology Practice, vol. 21, no. 3, pp. 265-267, 2010.

[70] W. Zhao, "The clinical research of shenqi fuzheng injection combined with TC theraputic regimen for the treatment of advanced ovarian epithelial carcinoma," Journal of Chinese Physics, vol. 35, no. 27, pp. 22-26, 2012.

[71] M. Y. D. Zhao, N. C. Quan, and W. X. Quan, “The theraputic observation of shenqi fuzheng injection combined with docetaxel and cisplatin for the treatment of advanced cervical cancer," Practical Clinical Journal of Integrated Traditional Chinese and Western Medicine, vol. 13, no. 3, pp. 3-5, 2013. 


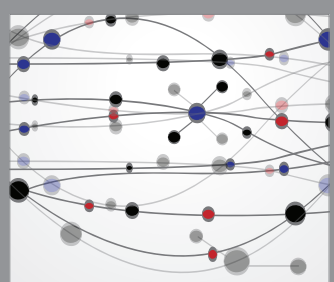

The Scientific World Journal
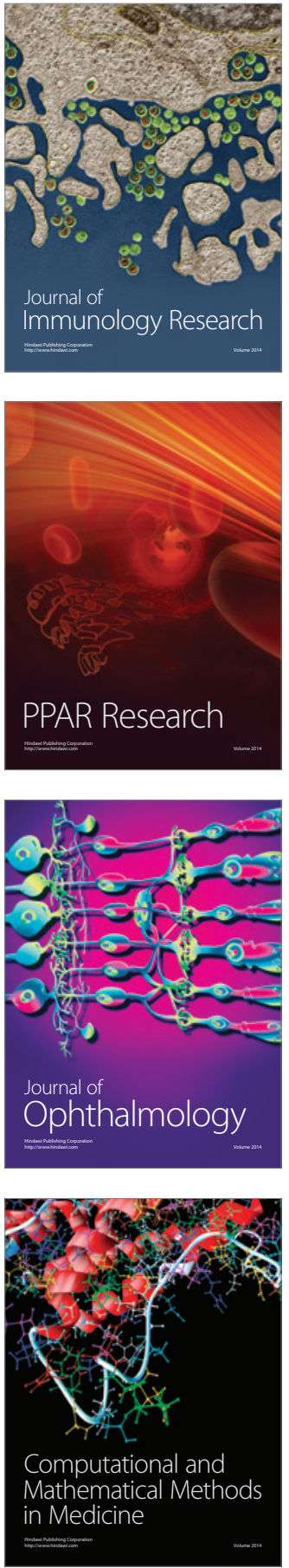

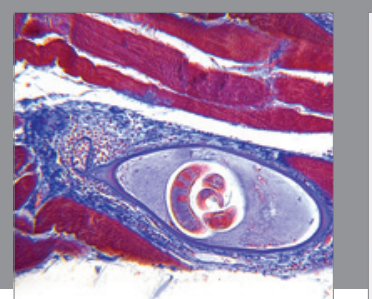

Gastroenterology Research and Practice
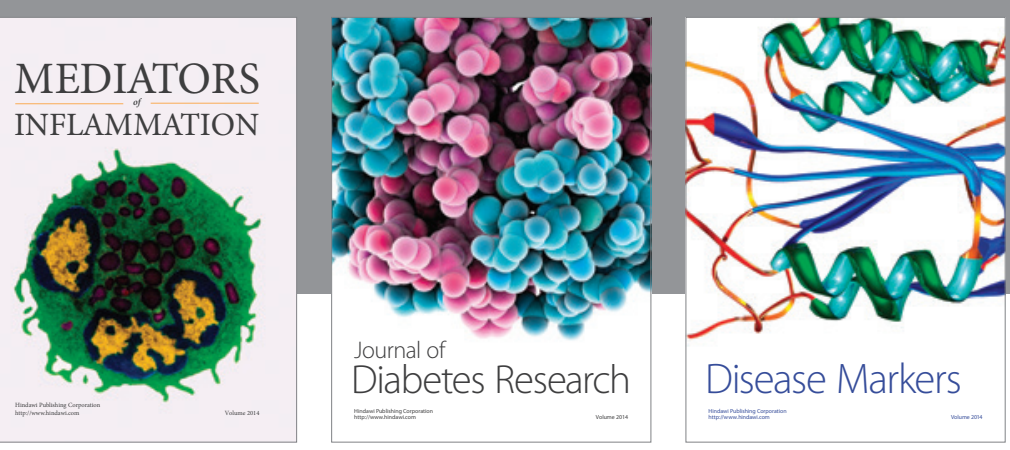

Disease Markers

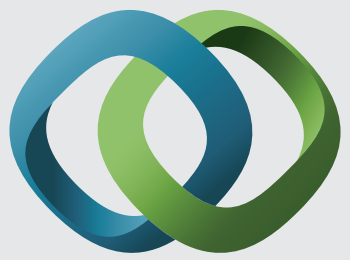

\section{Hindawi}

Submit your manuscripts at

https://www.hindawi.com
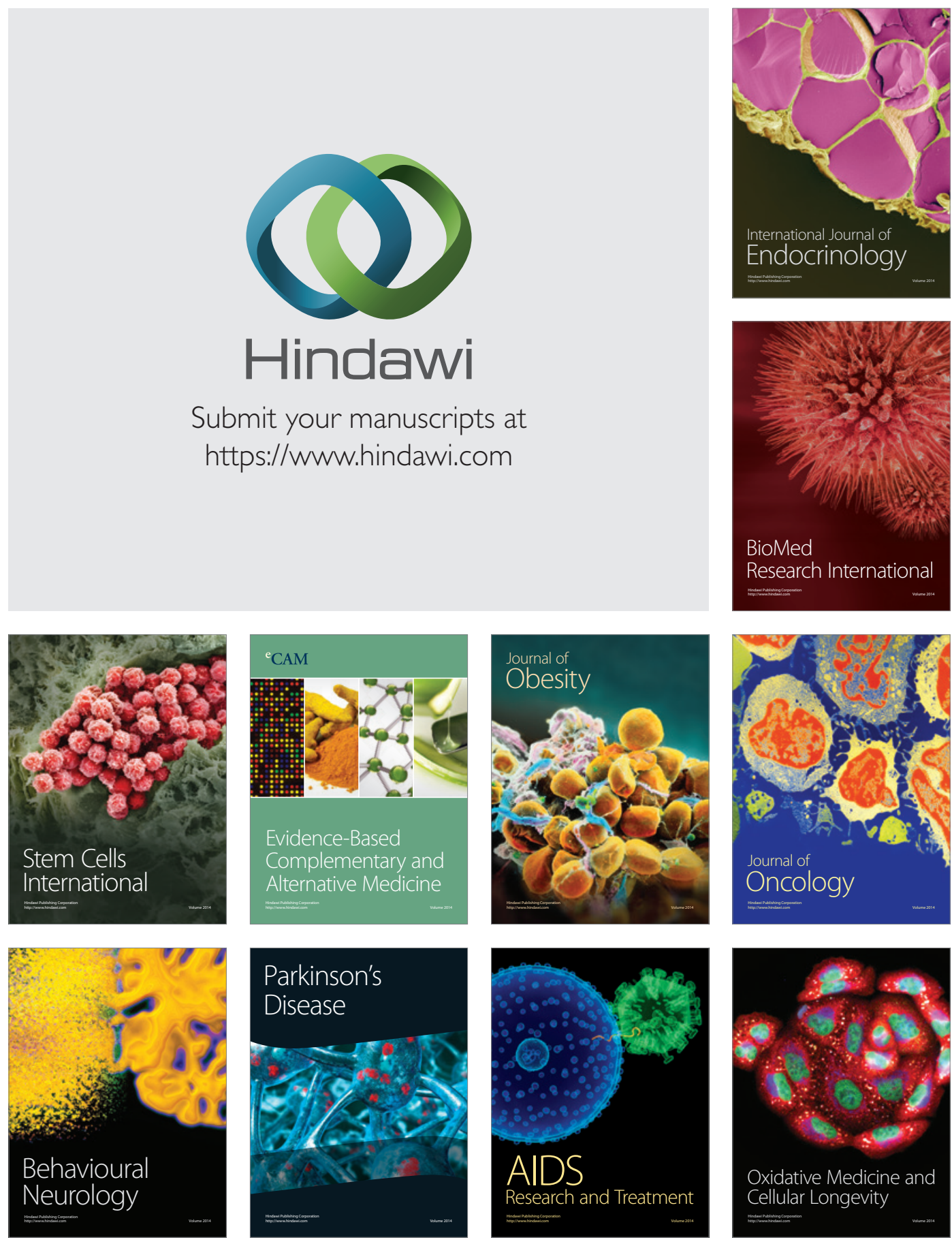